\title{
Statistical analysis of thermospheric gravity waves from Fabry-Perot Interferometer measurements of atomic oxygen
}

\author{
E. A. K. Ford ${ }^{1,2}$, A. L. Aruliah ${ }^{1}$, E. M. Griffin ${ }^{1}$, and I. McWhirter ${ }^{1}$ \\ ${ }^{1}$ Atmospheric Physics Laboratory, Department of Physics and Astronomy, University College London, Gower Street, \\ London, WC1E 6BT, UK \\ ${ }^{2}$ British Antarctic Survey, High Cross, Madingley Road, Cambridge, CB3 OET, UK
}

Received: 20 October 2006 - Revised: 24 September 2007 - Accepted: 17 December 2007 - Published: 4 February 2008

\begin{abstract}
Data from the Fabry-Perot Interferometers at KEOPS (Sweden), Sodankylä (Finland), and Svalbard (Norway), have been analysed for gravity wave activity on all the clear nights from 2000 to 2006 . A total of 249 nights were available from KEOPS, 133 from Sodankylä and 185 from the Svalbard FPI. A Lomb-Scargle analysis was performed on each of these nights to identify the periods of any wave activity during the night. Comparisons between many nights of data allow the general characteristics of the waves that are present in the high latitude upper thermosphere to be determined. Comparisons were made between the different parameters: the atomic oxygen intensities, the thermospheric winds and temperatures, and for each parameter the distribution of frequencies of the waves was determined. No dependence on the number of waves on geomagnetic activity levels, or position in the solar cycle, was found. All the FPIs have had different detectors at various times, producing different time resolutions of the data, so comparisons between the different years, and between data from different sites, showed how the time resolution determines which waves are observed. In addition to the cutoff due to the Nyquist frequency, poor resolution observations significantly reduce the number of short-period waves $(<1 \mathrm{~h}$ period) that may be detected with confidence. The length of the dataset, which is usually determined by the length of the night, was the main factor influencing the number of long period waves $(>5 \mathrm{~h}$ ) detected. Comparisons between the number of gravity waves detected at KEOPS and Sodankylä over all the seasons showed a similar proportion of waves to the number of nights used for both sites, as expected since the two sites are at similar latitudes and therefore locations with respect to the auroral oval, confirming this as a likely source region. Svalbard showed fewer waves with short periods than KEOPS data for a season when both had the same time resolution data. This
\end{abstract}

Correspondence to: E. A. K. Ford

(eakf@bas.ac.uk) gives a clear indication of the direction of flow of the gravity waves, and corroborates that the source is the auroral oval. This is because the energy is dissipated through heating in each cycle of a wave, therefore, over a given distance, short period waves lose more energy than long and dissipate before they reach their target.

Keywords. Atmospheric composition and structure (Airglow and aurora) - Meteorology and atmospheric dynamics (Thermospheric dynamics; Waves and tides)

\section{Introduction}

The Atmospheric Physics Laboratory (APL) at University College London (UCL) has a network of Fabry-Perot Interferometers (FPIs) located in northern Scandinavia (see e.g. Aruliah et al., 2004). Atmospheric gravity waves (AGW) have been detected in FPI measurements of the $630.0 \mathrm{~nm}$ atomic oxygen by Ford et al. (2006) and Ford et al. (2007). This emission occurs at an altitude of $240 \mathrm{~km}$, and therefore these gravity waves are in the high-latitude upper thermosphere. Gravity waves at lower altitudes mostly have their origin in the troposphere. They are formed from, for example, thunderstorms or air rising over mountain ranges. Amplitudes of the waves increase with decreasing density at increasing altitudes. The gravity waves that are formed in the troposphere have mostly dissipated long before they reach F-region altitudes.

Gravity waves observed in the FPI data therefore have to be created in situ in the thermosphere. The mechanisms to create these gravity waves are auroral in origin. Large-scale gravity waves are thought to be generated in magnetic storms by one of two mechanisms. Particle precipitation in auroral regions will create localised heating that could set off waves, as could the Lorentz forces and Joule heating from electrojet currents (de Deuge et al., 1994). The theory of

Published by Copernicus Publications on behalf of the European Geosciences Union. 
gravity wave behaviour and the expected properties of the waves have been reviewed by Hunsucker (1982) and Hocke and Schlegel (1996). Variations in the neutral atom emission intensities, neutral temperatures and winds have been detected, that have been caused by atmospheric gravity waves and were described in Ford et al. (2006) for a case study of 25 November 2003 (and see Sect. 3.1). Waves caused by both of these mechanisms were determined.

Atmospheric gravity waves are an important mechanism for energy and momentum transfer in the atmosphere (see e.g. Williams et al., 1993, and the review by Hocke and Schlegel, 1996). However, due to the difficulty in measuring the thermosphere, the majority of measurements of AGWs have been through their ionospheric counterpart, Travelling Ionospheric Disturbances (TIDs). These are often linked to AGWs, but the exact relationship is not well understood. It is therefore important to make independent measurements of the thermospheric AGW to properly ascertain their properties and behaviour. Gravity waves have also been observed in the upper thermosphere over the southern polar cap, for example, by de Deuge et al. (1994) and Innis et al. (2001) with photometer observations of the $630.0 \mathrm{~nm}$ oxygen emissions and by Innis and Conde (2002) in satellite data. Innis and Conde (2001) observed gravity waves in vertical thermospheric winds from the Dynamics Explorer 2 (DE2) satellite.

In depth studies of individual gravity wave events are useful to ascertain information on the origin and source of gravity waves, and to determine their properties. However, from only a few nights of data, it is not certain whether the waves seen are the usual behaviour of the thermosphere, or whether there are atypical conditions. Therefore, the data presented in this paper makes use of the large database of FPI measurements available from operating the instruments at various sites over many years. This can be used to statistically analyse the data to determine how frequently gravity waves are present, what periods they have, and what factors influence their amplitude or frequency of occurrence.

The FPIs have been operating routinely most winters for many years. Data availability from the various sites are summarised in Sect. 2. Studies that can be made with the datasets include differences between the sites, due to their geographical or geomagnetic locations (the KEOPS and Sodankylä sites are in the auroral oval area, while Svalbard is in the polar cap region). Also, for example, would the FPI with the higher time resolution data, due to a more sensitive detector, detect more waves than the other two sites with their lower time resolutions? There has not been much progress on inferring the climatology of global TIDs (Fritts and Alexander, 2003) because of the limitations of each observational data set, in terms for example of location, altitude and frequency range of observations, and period of the dataset. APL's FPI data is limited in the same way, in just observing one altitude in the high latitude Northern Hemisphere, during hours of darkness. The climatology of gravity waves within this limit is still of importance, however, and can provide significant new information. Data have been collected from solar maximum to solar minimum, so the dependence of the number of gravity waves formed on solar energy input to the thermosphere can be investigated with this data set. Additional solar effects through geomagnetic activity levels can be studied with all of the sites, by binning geomagnetically quiet, moderate, and active nights separately. Whether any particular periods are preferentially excited over others should be determinable by collating the periods of waves observed over a statistically significant number of nights. However, there are experimental limitations. The periods of the gravity waves observed are limited by the length of the data set, i.e. the length of the night, and of the time resolution of the data, so this will bias the results by excluding periods outside of this range.

\section{Data and analysis}

The UCL Fabry-Perot Interferometers measure the atomic oxygen red line emission at $630.0 \mathrm{~nm}$, which has a peak intensity at about $240 \mathrm{~km}$ altitude with a range of $\pm 50 \mathrm{~km}$ (Solomon et al., 1988). Temperatures and wind velocities of the neutral atmosphere are obtained as well as the intensity of the line emission. The FPIs look at a $1^{\circ}$ field of view at an elevation angle of $45^{\circ}$. UCL has three FPIs, which are currently located in the auroral oval in Sodankylä, Finland $\left(67.4^{\circ} \mathrm{N}, 26.6^{\circ} \mathrm{E}\right)$, and KEOPS (Kiruna Esrange Optical Site), Sweden $\left(67.9^{\circ} \mathrm{N}, 21.1^{\circ} \mathrm{E}\right)$, and in the polar cap at Longyearbyen on Svalbard $\left(78.2^{\circ} \mathrm{N}, 15.6^{\circ} \mathrm{E}\right)$ (e.g. Aruliah and Griffin, 2001). The number and location of the instruments has, however, varied over the period that APL have been running the FPIs. The first FPI was installed at Kiruna in 1980, which is only $\sim 50 \mathrm{~km}$ from the current site at KEOPS. Svalbard data are available from 1983 and Sodankylä data from 2002. The detectors on each of the instruments have changed several times during this time, and so therefore has the time resolution of the available data. For this study Kiruna and Svalbard data are taken from the winter seasons (typically September to April at Kiruna and Sodankylä), from 2000 to 2006. APL has a collaboration with the University of Lancaster (e.g. Kosch et al., 1997) which ran an FPI near Troms $\emptyset$ in Norway, at Skibotn $\left(69.3^{\circ} \mathrm{N}\right.$, $20.4^{\circ} \mathrm{E}$ ), which allows us to take tristatic measurements with three FPIs in northern Scandinavia.

As a result of the Fabry-Perot etalon, the image received from the detector is a series of concentric light and dark fringe interference rings. For a detailed discussion of FabryPerot interferometers, see Hernandez (1986), Born and Wolf (1987), and Hecht (2001). The intensity of the emission is given by the number of counts at the peak of a ring cross section. The temperature of the atomic oxygen atoms, and therefore of the thermosphere, is calculated from the width of the peak. The diameter of the interference ring is dependant on the Doppler shift and therefore gives the line of 
sight velocity. The FPI is scanned through different directions, to provide the different components of the wind vectors. However, as the image is all of the same $1^{\circ}$ portion of the sky, there is very little positional information in the rings, so nothing is lost by integrating around each ring and this also greatly improves the signal to noise ratio. The centre of the ring pattern is determined, and integrating around the circles produces a spectrum that has clear, sharp peaks that are used to determine the speed and temperature of the emitting oxygen atoms.

Data are taken in cycles, each instrument taking an exposure (from 20 to $60 \mathrm{~s}$, depending on the detector), looking at north, east, south, west, the zenith and a calibration lamp. Additionally, for KEOPS data before 2002 the northeast and north-west positions were viewed, to increase the data in the more active typical auroral oval area. When the Sodankylä FPI was installed, extra positions were viewed, at positions where both instruments can view the same volume of sky. The primary purpose of this was for co-located ion-neutral coupling studies made with the EISCAT radars, which are at the same locations as the FPIs. The results from this were presented in Aruliah et al. (2004, 2005), Ford et al. (2006). At Svalbard, in the polar cap region, the southeast and south-west positions are viewed, again to increase coverage towards the auroral oval, this time to the south of the site.

Cycle times vary depending on detector, but for example in the 2003-2004 winter season cycle times were $3.5 \mathrm{~min}$ for KEOPS, $8.5 \mathrm{~min}$ for Sodankylä, and $10.1 \mathrm{~min}$ for Svalbard. This gives a maximum detectable frequency (the Nyquist frequency) of 8.6 cycles per hour for KEOPS data. These cycle times in principle allow waves to be detected in the data of as short as 7-min periods for KEOPS or 17 for Sodankylä, i.e. twice the period of observations. The Brunt-Väisälä period, the minimum sustainable wave period of the atmosphere, is at this altitude $(240 \mathrm{~km}$ ) around $12 \mathrm{~min}$ (e.g. Hargreaves, 1979; Innis et al., 2001). Temporal resolutions as good as this are desirable for all data sets, so as to have sufficient sampling to be able to determine all waves down to $12 \mathrm{~min}$. The longest periods detectable are up to half the period of darkness, which in the polar winter night extends up to $24 \mathrm{~h}$ per day for over three months. In practice though, only periods less than $6 \mathrm{~h}$ are included, as periods longer than this are hard to distinguish from atmospheric tides. As the width of the $630.0 \mathrm{~nm}$ emission layer is approximately $100 \mathrm{~km}$ (Solomon et al., 1988), the FPIs will be sensitive to gravity waves with a vertical wave length greater than $100 \mathrm{~km}$ only. This means waves that are observable with the FPIs are limited to those with periods greater than about 26 min (Fritts and Hoppe, 1995).

The time series analysis performed on these data was a Lomb-Scargle least squares frequency analysis of unevenly sampled data as first formulated by Lomb (1976) and further developed by Scargle (1982). This method was used to cope with the uneven sampling of the data. This is created by the cycles of look directions, and other effects, such as the absence of data points, due to either cloud cover or the non-fitting of spectra due to poor signal to noise in the intensities. The application of this analysis to the FPI data is described in Ford et al. (2006). An additional advantage of the Lomb-Scargle analysis programme is that it normalises the time series using the mean of the data, which means that various data sets from different sites and under different conditions can be directly compared. Therefore, comparisons of different data sets, both with data from different instruments and from data from one instrument with different detectors can be made, as dependences of the analysis on background intensity levels will not affect the analysis.

Determining the proportion of nights where gravity waves are present will obviously be limited by the data available. The airglow and auroral emissions are only detectable over background solar emissions at night times; consequently data can only be collected during the autumn, winter and spring at the high latitudes of the instruments, inside the Arctic Circle. This will limit studies of the seasonal variability of gravity wave occurrence, as the day lit polar summer cannot be observed at all, and the lengths of the season and nights in the autumn and spring are much shorter than the continuous darkness of the winter polar nights. Fritts and Alexander (2003) report in their review of gravity waves a greater number of gravity waves in winter, and less in the summer, in the high latitude northern mesosphere using ionosonde and radar data. This effect, however, was observed at lower altitudes where the source of the gravity waves was likely to be tropospheric. Ogawa et al. (1987) also found that there was a maximum in gravity wave activity in winter and a minimum in summer in medium scale TIDs from satellite data. However, summer/winter effects can not be detectable with optical data as is used here. Ogawa et al. (1987) also found that the majority of the waves propagate equatorward, and that the number of waves detected did not increase with geomagnetic activity.

Data are not necessarily collected every night through the observing season, due to instrument failures, computer crashes, power cuts, and similar interruptions. When the instruments are running correctly and collecting data, the next obvious hindrance to collecting useable data is the amount of cloud cover. This significantly reduces the amount of data available for the statistical survey, and will bias the results. However, as tropospheric cloud cover does not influence the production or propagation of upper thermospheric gravity waves, there should be no systematic bias and the percentage of nights where gravity waves are observed should be representative of the whole observing season. Nights with total or partial cloud cover during the time the FPIs were running are excluded from the analysis.

Cloud cover levels are determined from a variety of sources, the simplest being all sky camera (ASC) keograms, when available, as these show auroral activity and cloud cover for a night. However, these are limited by a number 
of factors, such as earlier saturation detection levels for the detectors (particularly a problem at Svalbard, which is also shut down around the new moon time), and the keograms with green line $(557.7 \mathrm{~nm})$ filters only show auroral activity or cloud cover, and a quiet but clear night will be black. APL have a white light ASC at KEOPS, and individual images can be studied, and white light images are taken with the Sodankylä ASC. In addition to the ASCs, weather satellite images are available at various intervals on most nights. The infrared images from the European weather satellite METEOSAT provide night time weather information.

An alternative method is to use the FPI data itself to see if a night is cloudy. If the sky is overcast, the intensity peaks will coincide for all look directions. Some time series plots of line-of-sight wind data from opposite look directions (i.e. north and south, or east and west) have the same shape curves, but are mirror images of each other, for extended periods of time (i.e. hours). This is also usually indicative of cloud cover rather than an apparent divergent wind field, as this physically cannot be sustained for many hours. As clouds are between the $630.0 \mathrm{~nm}$ emissions and the FPI, the signal is scattered, and directional information is lost. The FPI measures winds from the Doppler shift on the $630 \mathrm{~nm}$ emission. For a simple case of a uniform wind over the whole field of view, a red shift in one look direction will be seen as a blue shift in the opposite direction. So plotting the lineof-sight winds against time from the two look directions will produce two lines that are mirror images of each other.

A total of 567 clear nights of data are available for this statistical analysis from the three sites of KEOPS (249 nights), Sodankylä (133) and Svalbard (185). Gravity waves are counted foe each site for each season (observing periods are at most from September to April, depending on location, instrument status and detector quality). These nights of data are divided into geomagnetic activity levels. Quiet is determined as having all 3 -hourly $K_{p}$ indices over the $24 \mathrm{~h}$ period of a night (from midday to midday on the following day) as being below 3 . Moderate has levels between 3 and 5 for the entire night, whereas active nights need to have a $K_{p}$ value greater than 7 at some point during the night. As the number of quiet nights is much greater than that of active nights, only the quiet nights have a sufficient statistical sample for any one observing season to be able to make statistically significant conclusions. An additional problem however with the geomagnetically quiet nights is that the signal to noise ratio is often very poor. The parameters, in particular the temperatures, cannot be sufficiently accurately determined, so these nights are also not included in this analysis. Therefore, to test for geomagnetic activity dependences, the whole database is used from all available years from each site. Many of the nights will not fit into these activity categories, as there will be a range of $K_{p}$ levels through the night, from quiet to moderate activity. Therefore, the total number of clear nights are separately analysed, to increase the statistical sample.
The Lomb-Scargle analysis is performed on each of the 567 nights of data to obtain the gravity wave periodicities. Only waves that are above the $70 \%$ confidence level are then used for the statistical analysis, which is determined from the length of the data set and the time resolution of the data. To view the data clearly for the many nights in each observing season, results are plotted in histograms of the occurrence of gravity waves for different periods. To plot data as histograms, the periods need to be binned. Bin sizes of $0.2 \mathrm{~h}$ (12 $\mathrm{min}$ ) are used, to give enough information on the period while still allowing sufficient waves in each bin. Periods up to $8 \mathrm{~h}$ are shown. Plots are therefore of the number of wave periods found in each period bin, and the tolerance on the periods is $0.2 \mathrm{~h}$.

Data are shown from all three sites: KEOPS, Sodankylä, and Svalbard, in the next three subsections (3.1-3.3), for the data available from 2000-2006. Not all seasons are shown, those with few clear nights of data over the winter are excluded, so that the most interesting seasons are discussed. Section 3.4 shows the data for these three sites summed up over all the years of data available during this period. This has the advantage of increasing the number of nights of data that are analysed, which increases the statistical sample, increasing weight to the results.

The figures in Sect. 3 show the peak periods in histograms for the intensities, winds, and temperatures. The various $K_{p}$ levels, as described above, are plotted together in separate colours. One count is shown for each period bin in a night, i.e. if there is a peak at any bin period in any of the look directions, then it is counted once, rather than a separate count for each look direction.

There is a general point to note about this type of plot. There is a bias to the periods found due to the way the data are collected. No very short periods will be seen, below twice the time resolution of the data, and there is a limiting value on the periods of the gravity waves due to the Brunt-Väisälä frequency (approximately $12 \mathrm{~min}$ ). However, once above a few tens of minutes, all the periods should be detected equally. There is a bias at the longer periods due to the maximum period observable being half the lengths of the data sets. Therefore, only waves up to this maximum limit have been included in these plots. Only the mid-winter months will have enough hours of darkness to include waves with periods up to $8 \mathrm{~h}$, while nights at the beginnings and ends of the observing seasons will have hours of darkness as short as four or five hours, depending on when the instruments are closed down for the summer. Thus the seasonal variation of the night will increase the proportion of shorter periods over the longer ones. In addition, as the shorter waves could have more wavefronts detected within the length of the night, and the longest may only see one cycle, the confidence of the detection will be greater for the short periods where the spectral power is reinforced by successive waves. 

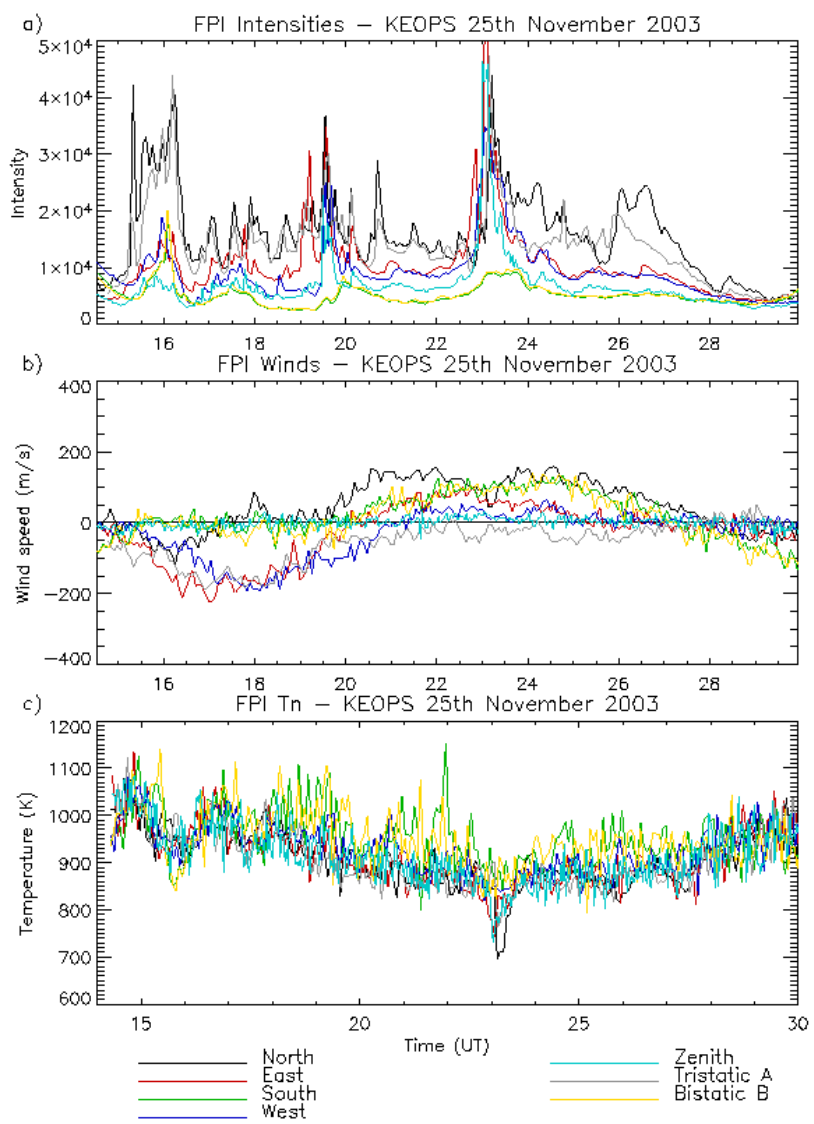

Fig. 1. $6300 \AA$ intensities (a), neutral winds (b), and temperatures (c) for all the look directions from KEOPS on 25 November 2003.

\section{Results and discussion}

\subsection{Case study}

Data from a case study of atmospheric gravity waves from 25 November 2003 were given in Ford et al. (2006). Gravity waves were seen in the atomic oxygen intensities and neutral temperatures from the FPI data from three instruments in mainland Scandinavia. The data from one of these sites, KEOPS, are shown in Fig. 1. The intensities (a), neutral winds (b) and temperatures (c) are shown in the three plots for each of the look directions, shown in colours. Periods of geomagnetic activity can be seen in the intensities, and the temperatures show a clear wave structure.

The Lomb-Scargle periodograms for these data are shown in Figs. 2 and 3. The intensities from the KEOPS data in Fig. 1 are shown in Fig. 2 along with the equivalent data from the other two sites, Sodankylä and Skibotn, which share a common volume (named the tristatic A point). The periodograms show the amplitudes of the waves against frequency. The period of the wave and the spectral power are also obtainable from the analysis, but amplitudes show relative strengths of the waves in relation to the background in-
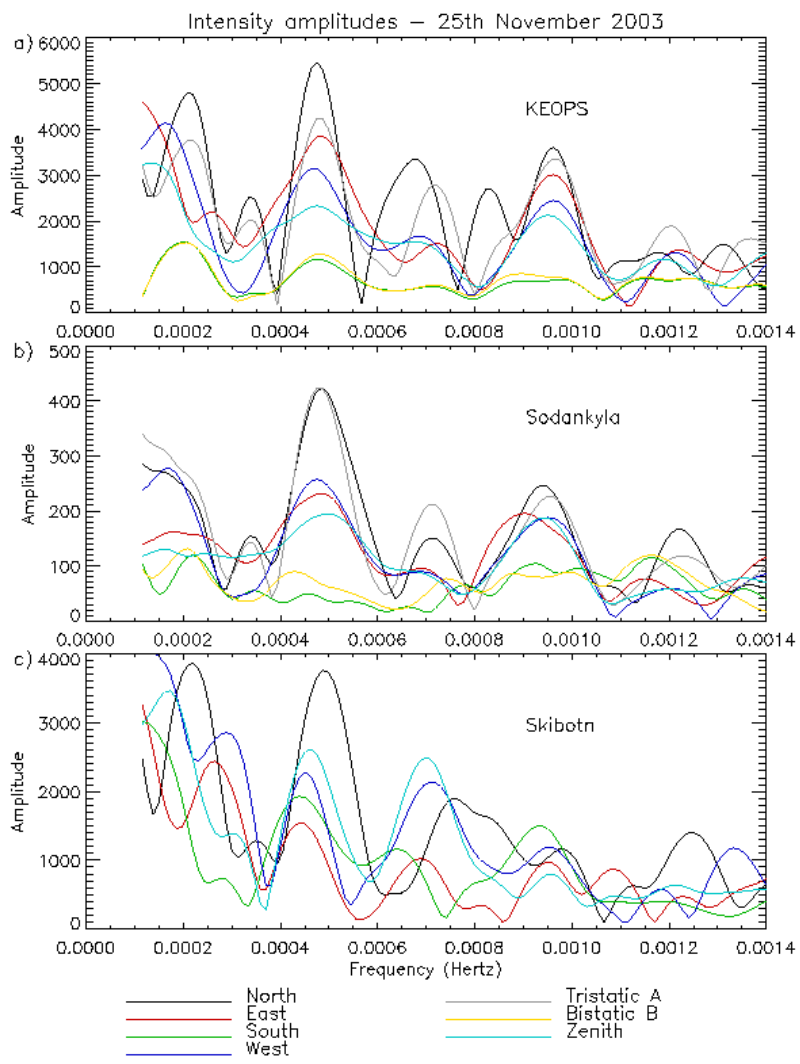

Fig. 2. Amplitudes of Lomb-Scargle periodograms for FPI intensities for (a) KEOPS, (b) Sodankylä, and (c) Skibotn, on 25 November 2003.

tensities, so waves between different look direction and instruments, with different time resolutions, can be directly compared. For both the $1.8-\mathrm{h}\left(9.7 \times 10^{-4}\right) \mathrm{Hz}$ and $3.7-\mathrm{h}$ $\left(4.6 \times 10^{-4} \mathrm{~Hz}\right)$ waves, KEOPS shows larger amplitudes in Fig. 2 to the north, which decrease towards the south, through tristatic A, east and west, zenith, and the smallest amplitudes are seen at the south and bistatic B positions. This is consistent with an auroral oval source region, which can be seen from magnetometer and all sky camera data for example, to be in the north throughout the night.

The relative sizes of the amplitudes between each of the look directions at Sodankylä are similar to those at KEOPS, which is at a similar latitude, and Sodankylä also has greater amplitudes to the north and A positions than the other look directions. The FPI intensities are not calibrated at present, so the intensity is dependant on detector sensitivity, so the intensities, and hence the amplitudes, are an order of magnitude smaller at Sodankylä than KEOPS.

The amplitude of the $3.7-\mathrm{h}\left(4.7 \times 10^{-4} \mathrm{~Hz}\right)$ wave is $12 \%$ of the maximum intensity at KEOPS and $11 \%$ for Sodankylä, so the wave amplitudes are comparable at both locations, as would be expected for sites at similar latitudes. The amplitudes of the 3.7-h wave in the south and bistatic B positions 


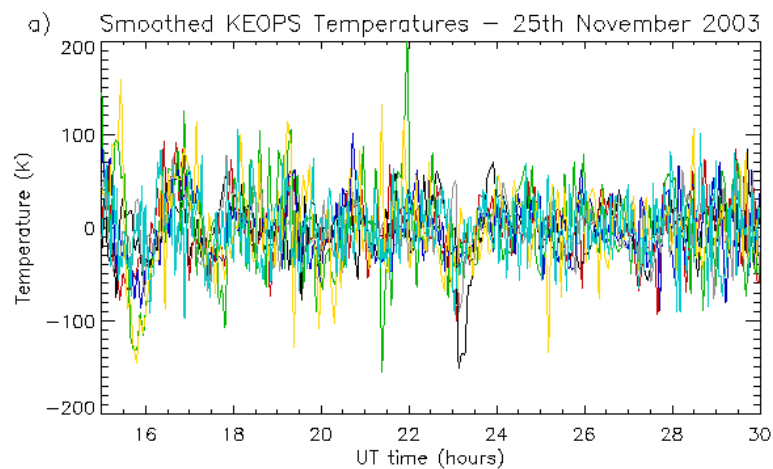

b) LSP: smoothed KEOPS Temperatures - 25th November 2003

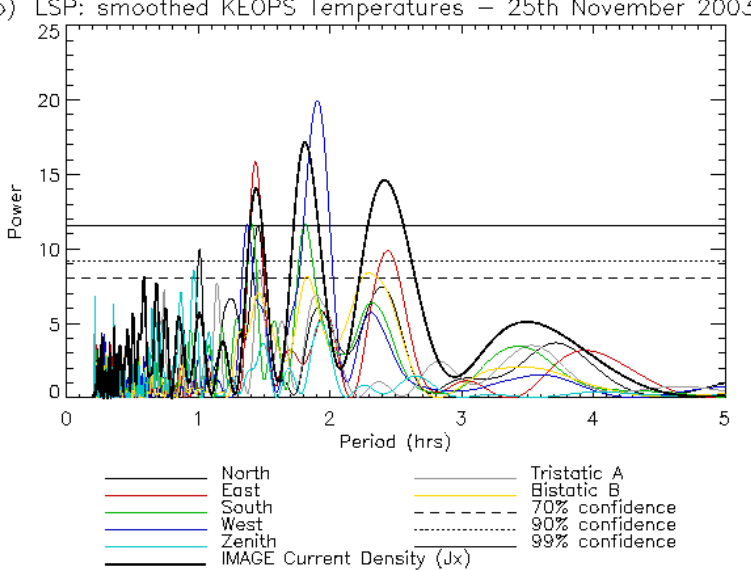

Fig. 3. Neutral temperatures with a 2-h smoothed value removed (a) and their periodogram (b) from KEOPS from 25 November 2003. The bottom plot also shows the periodogram for the equivalent current densities (thick black line) from IMAGE data (smoothed and reduced by a factor of 2 for comparisons).

are very small, but Skibotn sees larger amplitudes in all look directions for the 3.7-h wave. This is consistent with the auroral oval, which spreads across this whole region observed, but is stronger on this night across the northern half of the region, as can be seen from all sky camera data. Strong waves are also present at 2.5 and $3.7 \mathrm{~h}$ periods.

The top plot in Fig. 3 shows the temperatures for KEOPS on this night of 25 November 2003, but smoothed. Gradients are often seen over a night, as the temperatures are relatively slow to respond to changes in geomagnetic activity levels. This means that the majority of the power of the periodogram goes into this $24-\mathrm{h}$ period wave-like pattern. To compensate for this, the data is detrended by subtracting a 30-point running smoothing from the absolute temperature data, equivalent to high-pass filtering. This corresponds to an approximately 120 -min smoothing. This value is used as it removes the larger trends, and so removes power from the longest periods, those not associated with gravity waves, but is not so small as to remove power from the periods of interest. A wave structure can be clearly seen in the temperatures, and in all the look directions. The waves end at 04:00 UT,

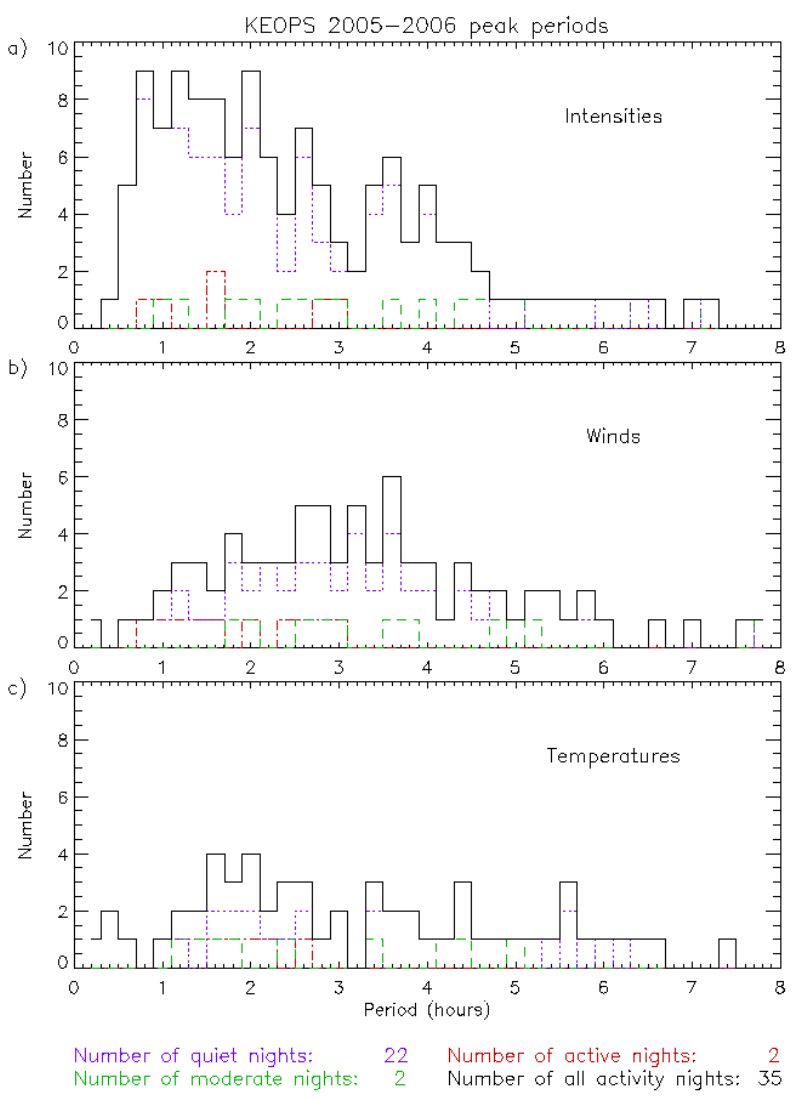

Fig. 4. Histogram of gravity wave periods for all nights and all activity levels, from KEOPS data over the winter season of 2005-2006 (solar minimum): (a) intensities, (b) winds, and (c) temperatures.

which is just due to the sky becoming overcast at this time. The Lomb-Scargle periodogram for this data is shown in the lower plot.

The periodicities in the different look directions are not quite as well defined as for the intensities, but are within the minimum error, of $4.5 \mathrm{~min}$ between data points. The most predominant periods are at $1.4,1.8$ and $2.3 \mathrm{~h}$, with other periods above the $70 \%$ confidence level at $1.0,1.1$ and $1.3 \mathrm{~h}$. The response times of the three parameters are different, and the same periodicities would not necessarily be expected in the winds as the intensities due to the complications of interactions between the gravity wave and the background wind field. Further details on the analysis of this night, including comparisons with data sets from other instruments, is given in Ford et al. (2006). Case studies from a high time resolution study of the KEOPS data from April 2004 is described in Ford et al. (2007).

\subsection{Time resolution effects}

Data are available from all three sites for the last season, the 2005-2006 winter, where the numbers of useable nights at each site although are not large, they are similar to each other 
and sufficient for analysis. Several results can be obtained with comparisons between these data sets. Additional information can be obtained for example from comparing the data between different sites from all the data available from all years, at each location. These data are shown in Sect. 3.7 below. Other studies are also presented in the following sections.

Data from KEOPS from the most recent season, 20052006 are shown in the histogram Fig. 4, as described in Sect. 2. Despite data being collected for seven months, there are relatively few nights of data available for this analysis compared with the number of nights of data collected. This is mostly due to poor weather conditions, as much of the winter was cloudy. This can also be seen in the Sodankylä and Svalbard data (below). The distributions of the number of waves with period follow similar patterns to the other data sets shown below. For example, there is an increase in the number of shorter period waves in the intensities, which is not seen in the other parameters. This is most pronounced for this data set, with the greatest number of waves between 0.7 and $2 \mathrm{~h}$. This is shown below to be due to the higher time resolution of this data set, which is due to a new more sensitive detector being installed at the beginning of the season.

Figure 5 shows the histogram for the 2005-2006 Sodankylä data. All being equal, the two sites should show the same results, as they are in reasonably close proximity and they are at similar latitudes. The proximity to solar minimum can clearly be seen in these data sets by the large proportion of geomagnetically quiet nights, which is over $70 \%$ of the total clear nights for this season. This plot can be compared with Fig. 4, which shows the KEOPS data for this same season. There are a comparable number of clear nights at each site, and the main difference between the two is the time resolution of the data sets. The KEOPS data at a cycle time of $3.5 \mathrm{~min}$, but Sodankylä had over twice this at $7.5 \mathrm{~min}$ resolution.

This difference can be seen in the plots in the intensities, as the Sodankylä data (Fig. 5a) have fewer waves at periods less than $2 \mathrm{~h}$ than the KEOPS data (Fig. 4a). The peak in the numbers of waves, i.e. the median period, are shifted to longer periods in the Sodankylä intensity data (Fig. 5a), with the mode period between approximately 2 and $5 \mathrm{~h}$, whereas the mode for KEOPS waves were between 1 and $4 \mathrm{~h}$. This is a bias in the measurements of the waves due to the poorer time resolution of the Sodankylä data, rather than any difference in the waves present at the two sites. The numbers of waves with periods greater than $3 \mathrm{~h}$ are comparable in each plot. The higher time resolution of KEOPS data allows a higher sampling rate of the waves, resulting in a more confident detection of the wave, and a higher spectral power. Therefore, more short period waves reach the $70 \%$ confidence level criteria and are included in these plots.

The winds from these two plots also show this time resolution effect, as there are fewer waves in the Sodankylä data (Fig. 5b) than the KEOPS data (Fig. 4b) with periods shorter

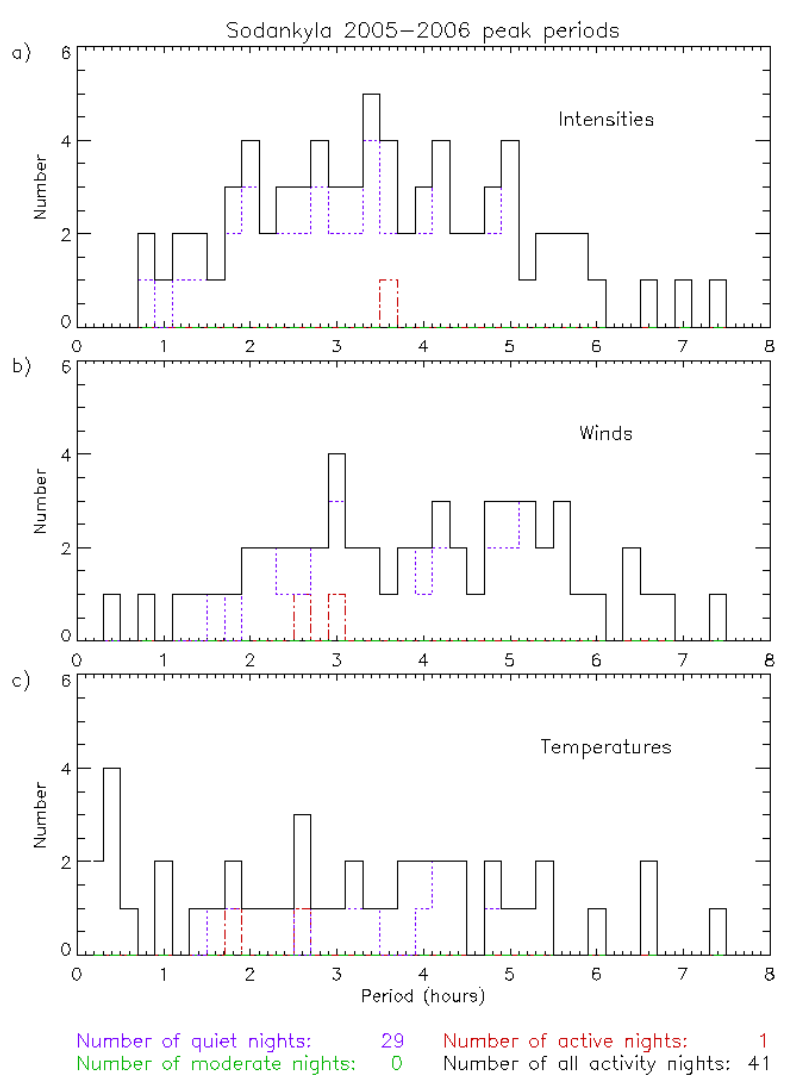

Fig. 5. Histogram of gravity wave periods for all nights from Sodankylä data over the winter season of 2005-2006: (a) intensities, (b) winds, and (c) temperatures.

than $4 \mathrm{~h}$. This is generally true of the temperatures too, with the shape of the distribution in Fig. 4c not seen in Fig. 5c. There are, however, four nights with waves of periods between 20 and $30 \mathrm{~min}$ in the Sodankylä data, which is greater than the number detected in the KEOPS data. However, for all of these waves, their power is only just over the $70 \%$ confidence level for the night, and they are only just over the minimum period detectable with the time resolution of the data, so they could be due to aliasing. The longer period waves, however, have similar distributions in the Sodankylä and KEOPS data for both the winds and the temperatures. This dependence on the time resolution of the data is also seen in other seasons of data, for example in the 2003-2004 season data, there are more available nights of data at Sodankylä than at KEOPS, but the Sodankylä FPI detects fewer waves than at KEOPS.

There are also differences between KEOPS (Fig. 4) and Sodankylä (Fig. 5)data at periods greater than two hours, but these are not as great as the short period wave numbers, and are enhanced by the different scales. This can be seen in Fig. 6, which shows the different number of gravity waves seen at KEOPS and Sodankylä for the 2005-2006 winter season. The large difference below around $2-3 \mathrm{~h}$ discussed 
KEOPS/Sodankyla comparisons 2005 - 2006 intensities

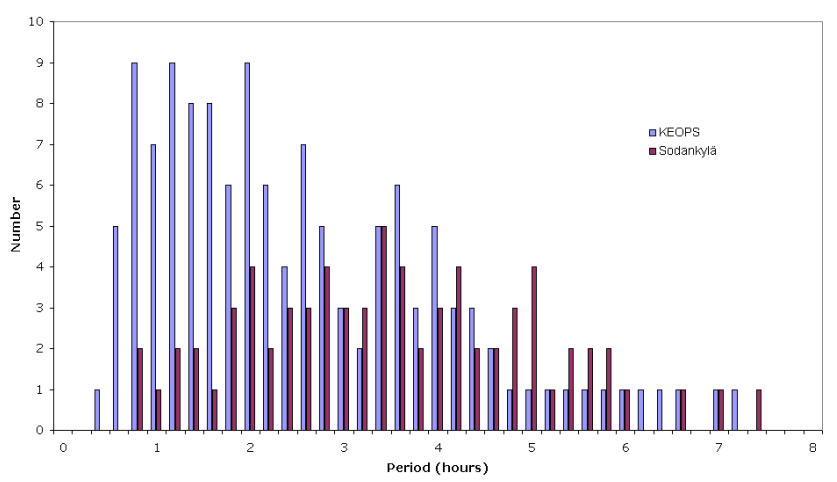

Fig. 6. Comparison of histograms of gravity wave periods for intensity data, from KEOPS and Sodankylä over the winter season of 2005-2006.

above can clearly be seen, but above this the two sites produce very similar numbers, with the greatest deviation being at $5 \mathrm{~h}$, with a difference of 3 . This is unlikely to be statistically significant.

\subsection{Parameter comparisons}

Data from 2004-2005 from KEOPS are shown in Fig. 7. In the intensities in Fig. 7a, the increased proportion of waves with shorter periods can be clearly seen. The distribution of the peaks in the wind data (Fig. 7b) is more evenly spread, and the majority of the waves have periods of $1-5 \mathrm{~h}$. The temperature data (Fig. 7c) shows a similar shape to the winds, but with fewer counts. For this season, $71 \%$ of all the clear nights were during the periods when there were 16 or more hours of darkness. All of the geomagnetically quiet nights were during this period, so for these dates all the waves up to $8 \mathrm{~h}$ should be detected.

There are a significantly larger number of waves seen in the intensities at shorter periods than longer, with the majority being between $1 \mathrm{~h}$ and $3 \mathrm{~h}$. The lower limit on this may be due to the time resolution of the data, but periods longer than $3 \mathrm{~h}$ are easily detectable, so this implies that longer period waves are not as common as short period waves. In comparison, the peaks in the winds and temperatures are more evenly spread across the period range, possibly with an increase in the numbers of waves with periods of a few hours, of around $2-5 \mathrm{~h}$.

The intensities are quicker to react to changes in conditions, such as gravity wave forcing from particle precipitation, than the winds and temperatures, due to the inertia of the thermosphere. This could explain the difference in the distributions of the periods in the different parameters, as the short period waves are not able to form in the winds and temperatures, and the inertia of the thermosphere in effect acts as a low band pass filter.

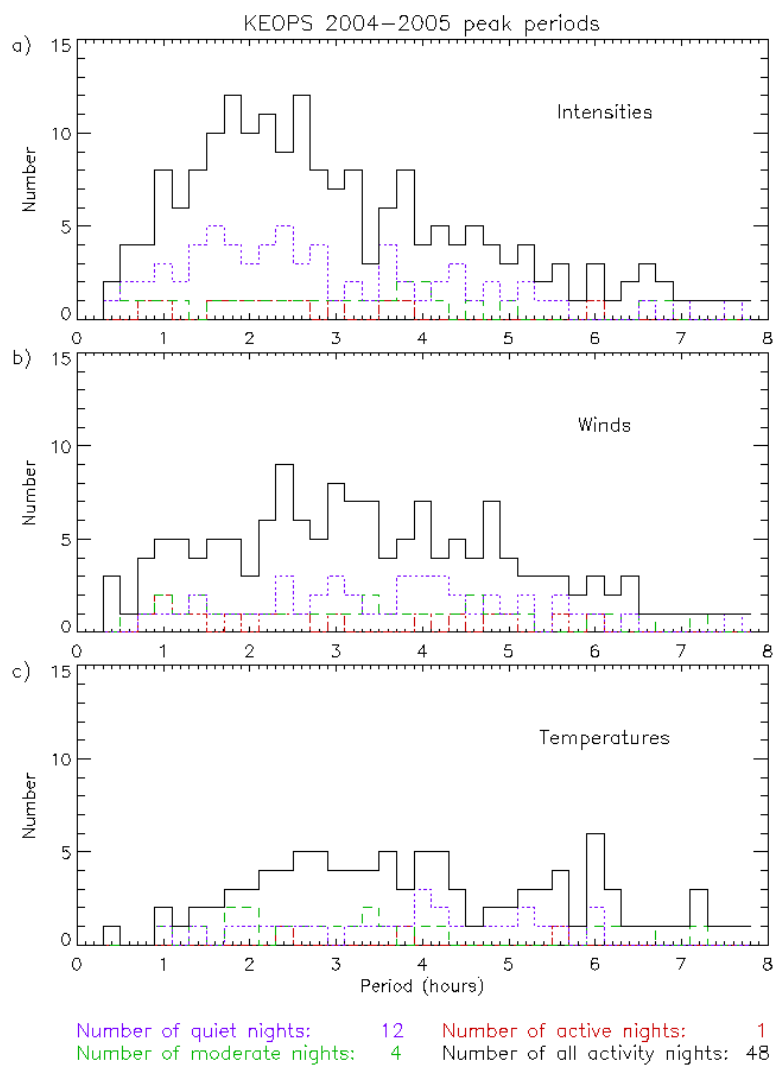

Fig. 7. Histogram of gravity wave periods for all nights and all activity levels, from KEOPS data over the winter season of 20042005: (a) intensities, (b) winds, and (c) temperatures.

\subsection{Geomagnetic activity dependences}

To increase the size of the statistical sample, the following plots show the results of adding the gravity waves found in each night over all available seasons between 2000 and 2006 for each site. There were a total of 249 nights used from the KEOPS FPI, 133 from Sodankylä, and 185 from Svalbard. Figure 8 shows a histogram of the gravity waves found in each period bin for all the KEOPS data from 2000 to 2006, in the same format as those for the individual seasons. The distributions of the waves over the period range are very similar to those of for example the 2004-2005 data in Fig. 7, for the intensities, winds, and the temperatures. The varying time resolution of the data over each of the years results in the number of the shortest periods of waves (less than an hour) being proportionally less than, for example, the 20052006 data in Fig. 4. The wind and temperature data again have broader peaks in the number distributions, and at longer periods than the intensities.

The main advantage of summing over all the years of data is that it increases the total number of nights with moderate geomagnetic activity (22 nights for KEOPS) and active nights (14). These are still not large statistical samples, but 
they are large enough to have more than one wave in each period bin, which was often the case with individual years of data. The only year with more than two active nights available was the 2000-2001 season, which was at solar maximum.

Figure 8 shows, particularly with the intensities, that the moderate and active nights have a similar distribution of periods to that of the quiet nights, with a peak between approximately 1 and $4 \mathrm{~h}$. This is in agreement with the individual seasons' histograms and for example results from Ogawa et al. (1987) to indicate that the distribution of gravity wave periods is independent of geomagnetic activity. As the Sodankylä FPI was not installed until 2002, past the solar maximum, and there were roughly half the total number of nights available compared to KEOPS, there were only two active nights for the total of the Sodankylä data, shown in Fig. 9. The moderate geomagnetic activity nights also only produce one or two waves in any one period bin, so also cannot be used to draw any useful conclusions.

The total of the four seasons of Sodankylä data for all activity levels produce reasonable numbers of gravity waves. Few waves are observed with periods less than roughly $2 \mathrm{~h}$, which again is due to the poorer time resolution of the data, which was on average 3 to 4 times longer than for KEOPS. The highest number of waves in any one period bin in the intensities at Sodankylä in Fig. 9a is 12 (at $3.4 \mathrm{~h}$ ), whereas for KEOPS, in Fig. 8a the highest number is 35 (at $2.0 \mathrm{~h}$ ). Sodankylä therefore has only $35 \%$ of the peak number of waves of KEOPS, but it has 54\% the total number of nights. However, as the peak at Sodankylä is shifted due to the reduced number of short period waves due to the lower time resolution, the numbers at the same period bin (e.g. $3.4 \mathrm{~h}$ ) should be compared (this value is used as it is not in the range where time resolution or night length effects are important, averages are discussed below). This gives approximately 52\% $( \pm 15 \%)$ of the KEOPS value at $3.4 \mathrm{~h}$, which is similar to the proportion of nights that were used for each data set. This is as would be expected, as the two sites are at similar latitudes, so are in similar parts of the auroral oval. They would therefore be expected to observe the same waves, especially as they have overlapping fields of view, within the experimental limits of the instrumentation.

\subsection{Spatial distribution of gravity waves}

Figure 10 shows the 2005-2006 season at Svalbard, after the Andor detector was installed at the beginning of December 2005. Cloud cover levels were considerable for this season, but despite the low number of clear nights available (22), and the proximity to solar minimum, a reasonable number of waves were detected, with up to 6 waves in any one period bin. This is most likely due to the improved time resolution of the data with the Andor detector, as cycle times of less than 4 min were achievable, which is comparable with

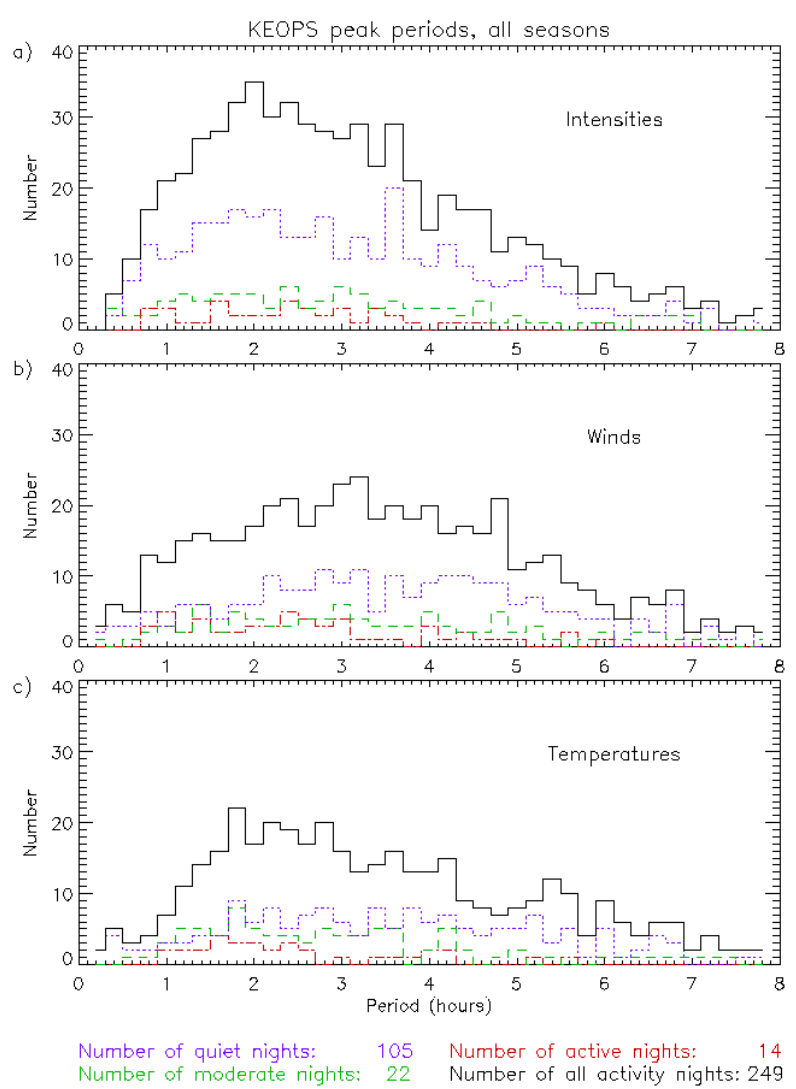

Fig. 8. Histogram of gravity wave periods for all KEOPS data (2000-2006) at all activity levels: (a) intensities, (b) winds, and (c) temperatures.

the KEOPS FPI, which also had an Andor detector for this season.

For the intensities at KEOPS in the 2005-2006 data (Fig. 4), the majority of the waves have periods between $0.5-$ $4.5 \mathrm{~h}$. However, for the Svalbard 2005-2006 data (Fig. 10), the majority of the waves have periods between 2 and $5 \mathrm{~h}$. The maximum number of waves in any one period bin is nine for KEOPS but six for Svalbard, but this could be explained by the fewer number of nights of data (22 in Svalbard compared with 35 at KEOPS), as well as the broader spread of waves across the period range.

There is only one wave with a period of less than an hour at Svalbard for this season, compared with 15 for KEOPS. As the time resolutions of the data sets are similar due to the same quality detectors, and the data sets are from the same year and so same point in the solar cycle, the main other difference between the sites is their location.

As high latitude thermospheric gravity waves are thought to have a source in the auroral oval, and as KEOPS is located in the nominal auroral oval, it would be expected to be able to detect all waves that are formed there. In contrast, Svalbard is at a higher latitude, in the polar cap region. Waves 

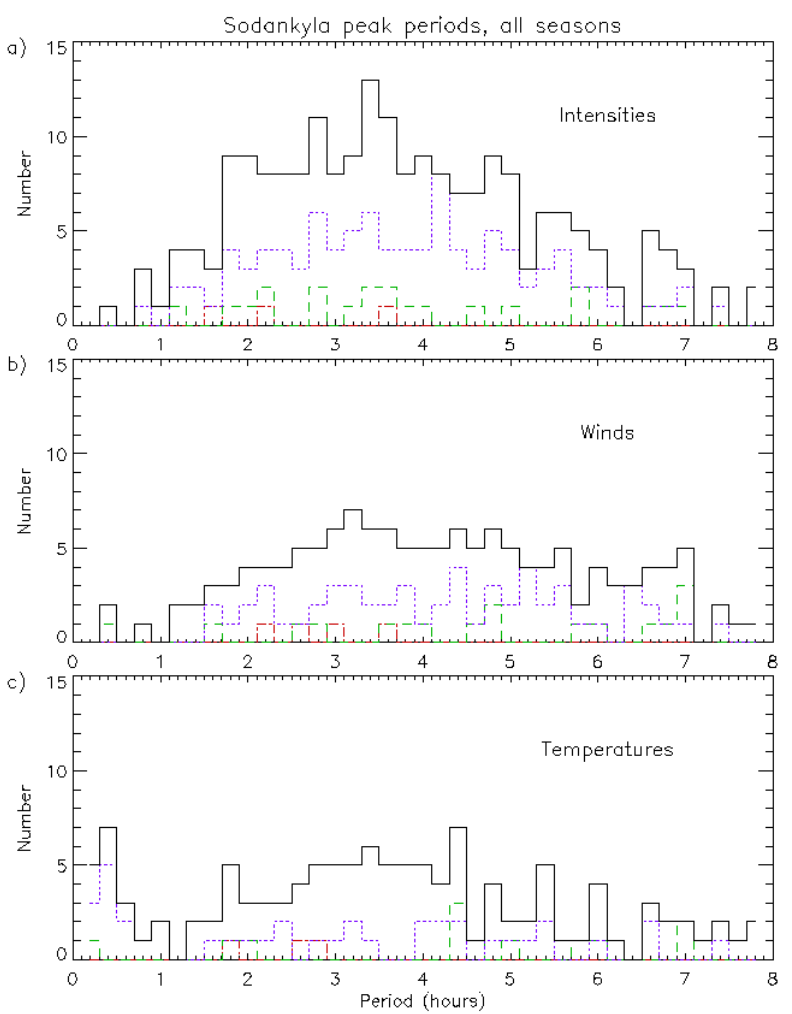

$\begin{array}{lll}\text { Number of quiet nights: } & 48 & \text { Number of active nights: } \\ \text { Number of moderate nights: } & 10 & \text { Number of all activity nights: } 133\end{array}$

Fig. 9. Histogram of gravity wave periods for all Sodankylä data (2002-2006) at all activity levels: (a) intensities, (b) winds, and (c) temperatures.

therefore have to travel some distance before being observed over Svalbard (there are over $1000 \mathrm{~km}$ between the Svalbard FPI and the mainland sites).

As the power and amplitude of a wave is dependant on frequency (e.g. Press and Rybicki, 1989; Hocke, 1998), waves with short periods will be attenuated more and they will therefore dissipate more quickly and over shorter distances than longer period waves. Short periods cover more cycles over a given distance than long periods so dissipation effects act over further distances. This is the same effect as the Qfactor in for example electromagnetic waves in circuits or seismic waves. This accounts for the reduced number of short period waves seen in the Svalbard data in relation to the mainland data. This effect has also been seen between the 2001-2002 data sets for KEOPS and Svalbard, which again had similar time resolutions, though slower at around 15-min cycle times.

The Svalbard data also show many more waves with longer periods than the KEOPS data, especially over $5 \mathrm{~h}$. This again is likely to be due to the timing of the data rather than a real variation in the gravity wave parameters. The majority of the nights used for the Svalbard data set were in

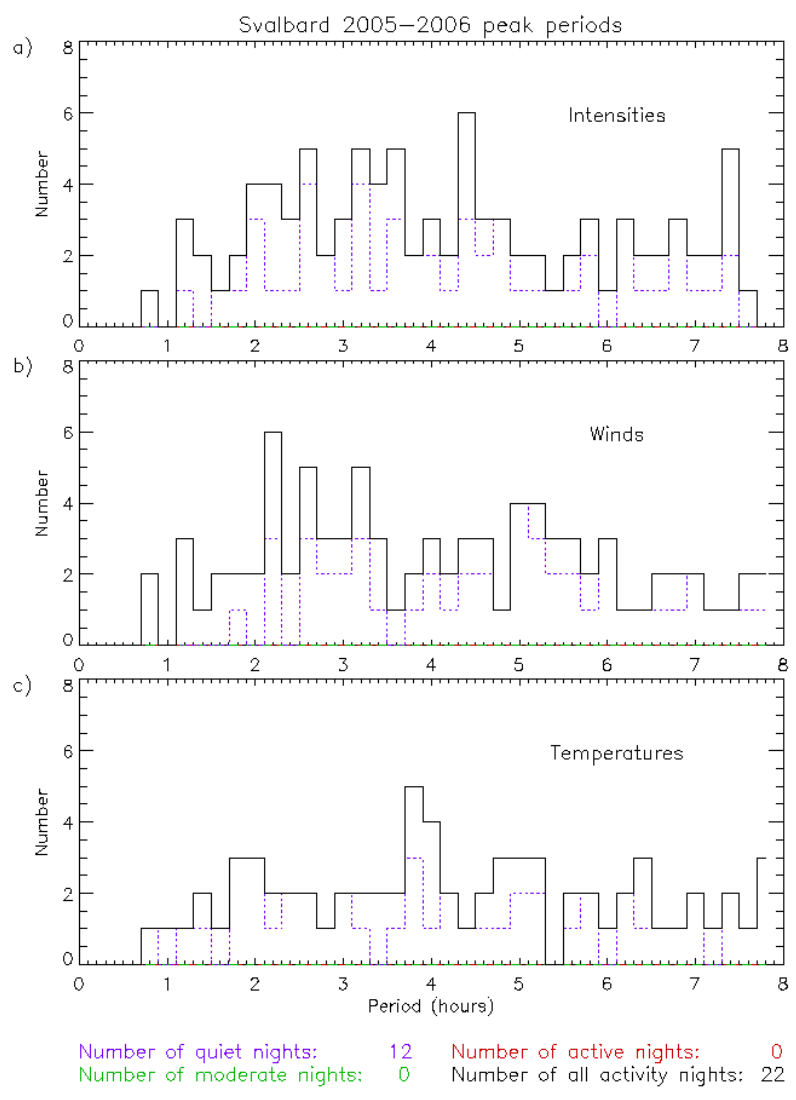

Fig. 10. Histogram of gravity wave periods for all nights and all activity levels, from Svalbard data over the winter season of 20052006: (a) intensities, (b) winds, and (c) temperatures.

December and January, whereas the KEOPS data was from the end of August through to March, and the long winter nights were mostly cloudy, with the majority of the clear nights in February and March. This would have resulted in a greater number of nights in Svalbard with long enough hours of darkness to observe the long period gravity waves than in KEOPS.

The waves in the Sodankylä data show a distribution more similar to the Svalbard data (Fig. 10) than the KEOPS data (Fig. 4), in all of the three parameters. This shows that the dissipation of short period waves can have the same consequences for the detection of gravity waves as a decrease in time resolution. Good quality detectors are therefore an important factor in obtaining a true distribution of gravity waves from the FPI data.

Figure 11 shows the total of all the waves from the Svalbard FPI over the five seasons of data from 2000 to 2006. The most obvious difference between this plot and the two for KEOPS (Fig. 8) and Sodankylä (Fig. 9) is the increased number of waves with long periods, due to the increased number of nights with $24 \mathrm{~h}$ of darkness at Svalbard at its higher latitude. This can be seen in the intensities, winds, 

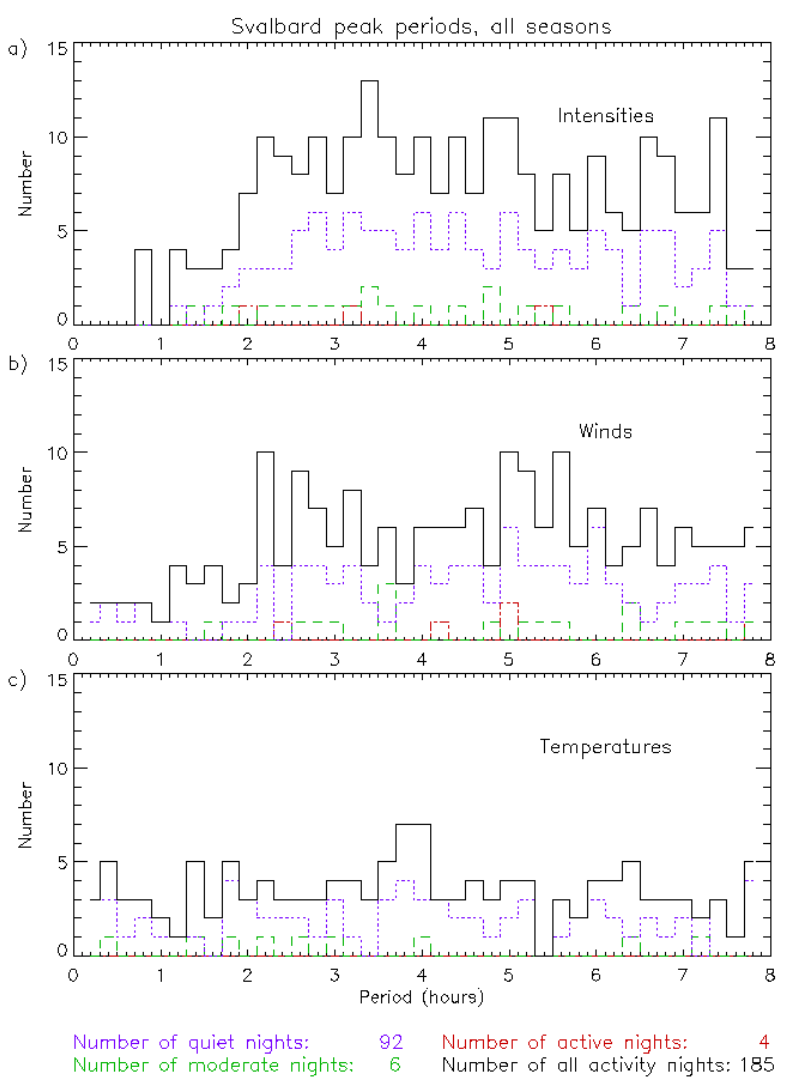

Fig. 11. Histogram of gravity wave periods for all Svalbard data (2000-2006) at all activity levels: (a) intensities, (b) winds, and (c) temperatures.

and temperatures. The range of time resolutions of the data over different seasons at Svalbard is similar to that at Sodankylä. As a consequence there is a reduced number of waves with periods below $2 \mathrm{~h}$ that is similar to Sodankylä. However, there is also a contribution from the 2005-2006 Svalbard database when the reduced number of short period waves is proposed to be due to the preferential dissipation of short period waves emanating from the auroral oval and travelling polewards.

The average occurrence per nights of gravity waves can be calculated from the total number of waves, over the period range used here, divided by the total number of clear nights used in the analysis, over all the seasons. The results of this for the three sites, for each parameter, are shown in Table 1.

The highest proportion of waves over the number of nights was in the KEOPS intensity data, with an average proportion of 2.5 waves per night detected. There are slightly fewer waves in the winds and temperatures. These numbers reflect the differences in different parameters, and the speed with which the intensities can respond to precipitation and energy input compared with the inertia that has to be overcome to move or heat the thermosphere. The occurrence of waves in
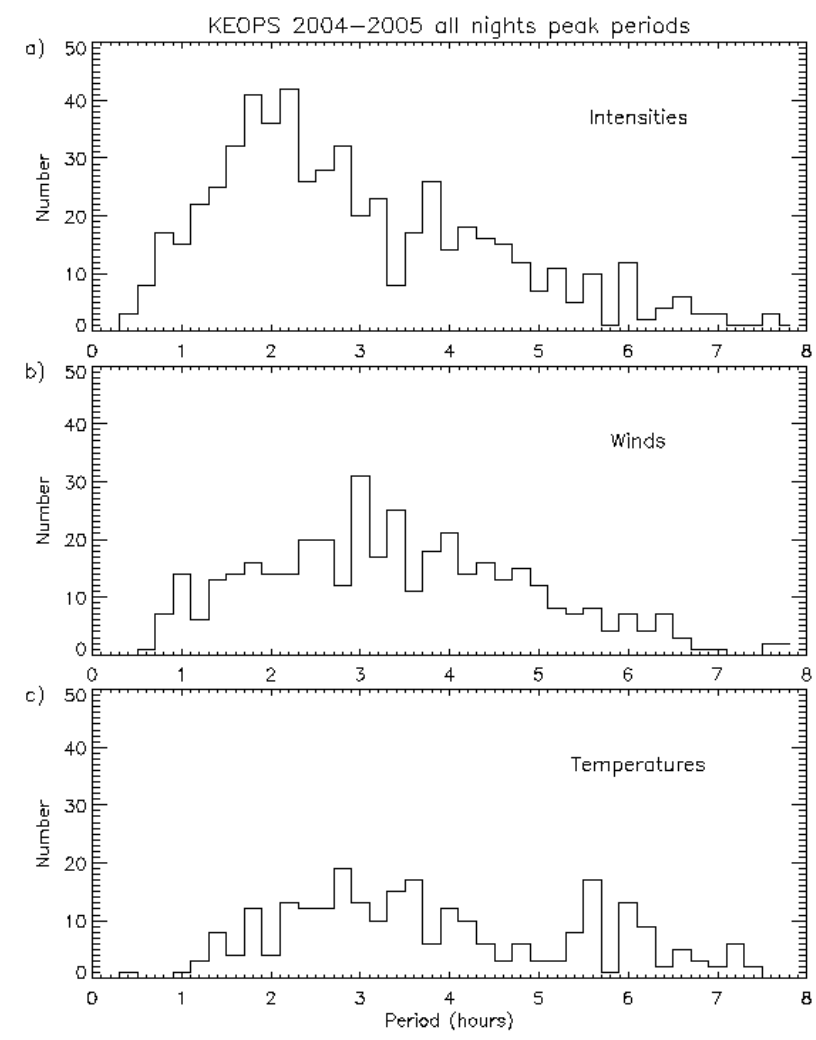

Fig. 12. Histogram of gravity wave periods for all nights from KEOPS data from the north, east, south, and west directions, over the winter season of 2004-2005: (a) intensities, (b) winds, and (c) temperatures.

the Sodankylä data is just over half as large as the KEOPS data. Svalbard data produce similar proportions to the Sodankylä data. This lower detection rate is most likely due to the lower time resolution of the data not sampling the waves sufficiently enough to give a spectral power over the $70 \%$ confidence level used here. Sodankylä and Svalbard data sets have on average approximately the same time resolutions, until the 2005-2006 season when the Svalbard detector was upgraded. The reduced proportion of short period waves at Svalbard in this last winter can no longer be attributed to the sampling time and therefore is interpreted as a real reduction. This difference could be attributed to the site locations. Svalbard is further from the typical source region of the gravity waves in the auroral oval, and so more waves would have dissipated by the time they have travelled the distance to the polar cap.

\subsection{Gravity wave propagation directions}

Figure 12 shows a reassessment of the 2004-2005 KEOPS season, by counting the gravity waves seen in each look direction. This can be used to show an indication of the proportion of nights where the gravity waves are seen in multiple 


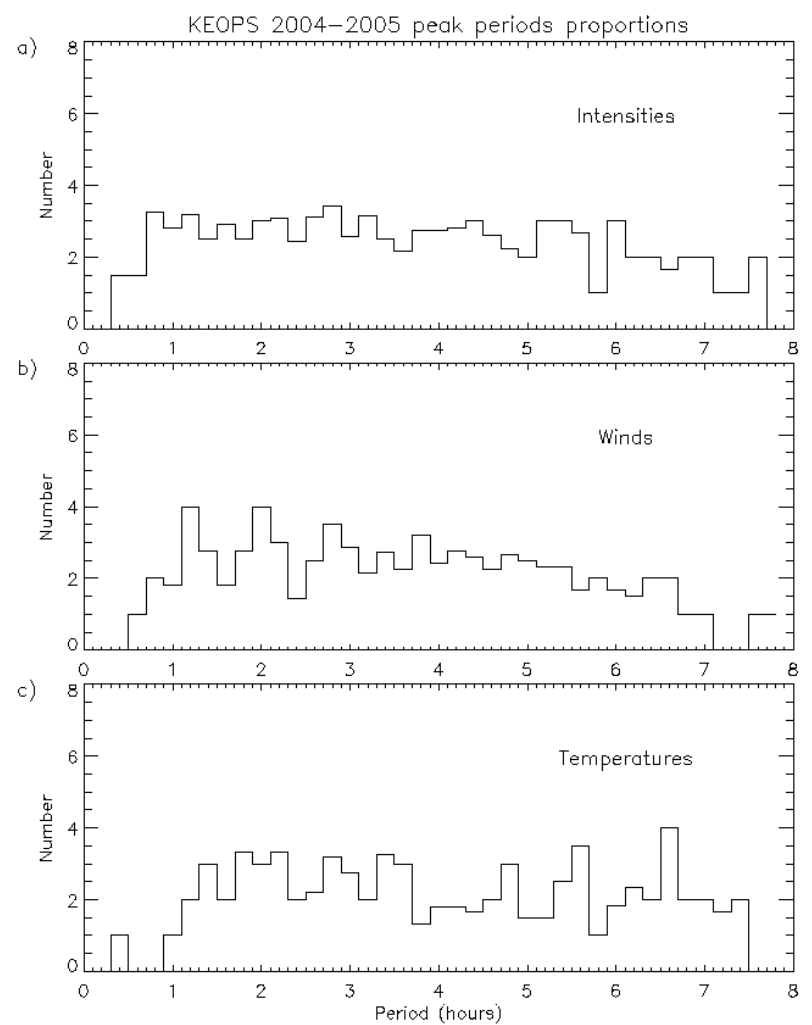

Fig. 13. Proportion of gravity wave periods for the north, east, south, and west directions compared with the number per night, from KEOPS data over the 2004-2005 season: (a) intensities, (b) winds, and (c) temperatures.

look directions. However, this will exaggerate the apparent number of gravity waves, so is only used for this study. Only the north, east, south, and west data are used, as if all the directions are included there will be a bias as they are not uniformly distributed over the region (the auroral oval is sampled more frequently through additional viewing volumes). The distribution of the waves with period has a similar shape to that of Fig. 7 where a gravity wave seen in several look directions within a cycle of observations is counted as only one gravity wave. In addition to this shape though, there are an anomalously increased number of wave peak periods at $6.0 \mathrm{~h}$ in the intensities and at 5.6 and $6 \mathrm{~h}$ in the temperatures. The peak at $5.6 \mathrm{~h}$ is due to the wave in the active night being observed in all the look directions. The 6-h wave, in intensities and temperatures is likely to be due to atmospheric tides, caused by solar heating, at a harmonic of the 24-h day, or to a harmonic of the two-cell convection pattern. This tide is probably not observed in the wind data due to the interaction of the tide with the background wind fields, for example from the two cell convection pattern. The tide would be expected to be stronger in the temperatures as it is caused by solar heating.
Comparisons in the proportion of counts over the periods of waves between the histograms in Fig. 12 and those in Fig. 7 will show an indication of the proportion of nights where the gravity waves are seen in multiple look directions. The general shape of the histogram in Fig. 12 and the total nights (black line in Fig. 7) are the same, though the count rate is approximately $25 \%$ of the plot counting each look direction separately. By finding the ratio of these two plots, the average number of look directions in which a wave is observed can be found. This is plotted in Fig. 13.

Apart from the decreases at very short and long periods, the distributions in Fig. 13 are fairly uniform. The average, from periods between 0.6 and $7.0 \mathrm{~h}$ are 2.6 for the intensities, 2.3 for the winds, and 2.2 for the temperatures. The standard errors on the means $(\varepsilon=\sigma / \sqrt{ } N$, where $\sigma$ is the standard deviation over $N$ data points) are $0.10,0.13$, and 0.17 , respectively. These averages mean that a wave is seen at a confidence level above $70 \%$ in over half of the four look directions (on average 60\%). From comparing with the case study on gravity waves in Ford et al. (2006), many of the strong waves that are seen with high confidence levels in one or two of the look directions, the waves are often detected in the other look directions but with smaller powers and lower confidence levels. If lower confidence levels were therefore to be considered, this number could be greater.

There are two possibilities for the waves not often being detected in all the look directions, with high powers. This could be an indication that the waves are only propagating across part of the field of view of the instrument, for instance along or across the auroral oval. Alternatively, the waves are dissipating before reaching all the look direction locations. To be able to detect the wave in one look direction with a high confidence level, the wave either needs to have a large amplitude over the background variations, or several wavelengths need to be observed. If the amplitude is large in one look direction, the wave is unlikely to have dissipated by reaching another look direction, which will only be a fraction of a wavelength away. Similarly, if several wavelengths are observed in one direction, the source is likely to be nearby and the wave should not dissipate over the distance covered by the field of view as this is less than the wavelength of the waves at this altitude. It is therefore more likely that the waves are not seen in some of the look directions due to the propagation angle of the wave. This could be an indication that the waves do not propagate uniformly away from the source, but have a preferred direction. From the results of the case study in Ford et al. (2006), this is likely to be in an equatorward or poleward direction, perpendicular to the auroral oval.

\subsection{Seasonal variations}

Data from the 2000-2001 winter season at KEOPS are shown in Fig. 14. This season was good both in terms of detector performance and cloud cover levels, consequently there were 
Table 1. Occurrence of gravity waves per night, for each site, over all seasons of data for the intensities, winds and temperatures.

\begin{tabular}{lcccccc}
\hline & \multicolumn{2}{c}{ KEOPS } & \multicolumn{2}{c}{ Sodankylä } & \multicolumn{2}{c}{ Svalbard } \\
& Total waves & Occurrence & Total waves & Occurrence & Total waves & Occurrence \\
\hline Intensity & 614 & 2.5 & 208 & 1.6 & 256 & 1.4 \\
Wind & 485 & 2.0 & 139 & 1.1 & 196 & 1.1 \\
Temperature & 387 & 1.6 & 121 & 0.9 & 135 & 0.7 \\
\hline
\end{tabular}

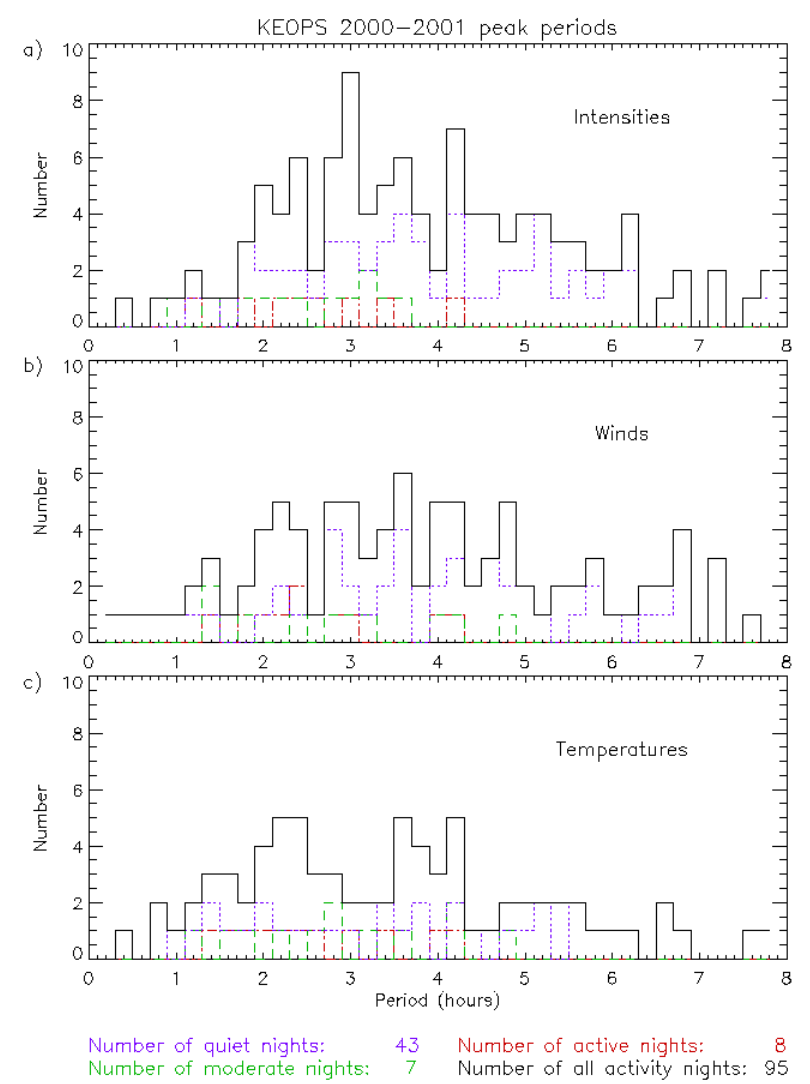

Fig. 14. Histogram of gravity wave periods for all nights and all activity levels, from KEOPS data over the winter season of 2000-2001 (solar maximum): (a) intensities, (b) winds, and (c) temperatures.

95 nights of data over the observing season. The distributions of the waves across the period range show the decrease in numbers of the shortest and longest period waves due, respectively, to the time resolution of the data set and the limited length of the data sets for some of the nights.

To better show the distribution of the waves across the year, and as this season has the most nights of data, the 20002001 KEOPS data are grouped together in months in Fig. 15. Only waves with periods up to $4 \mathrm{~h}$ are included, as otherwise the varying length of the night creates a bias towards the longer winter nights. The dotted line shows the number of nights of data that were used for each month. This
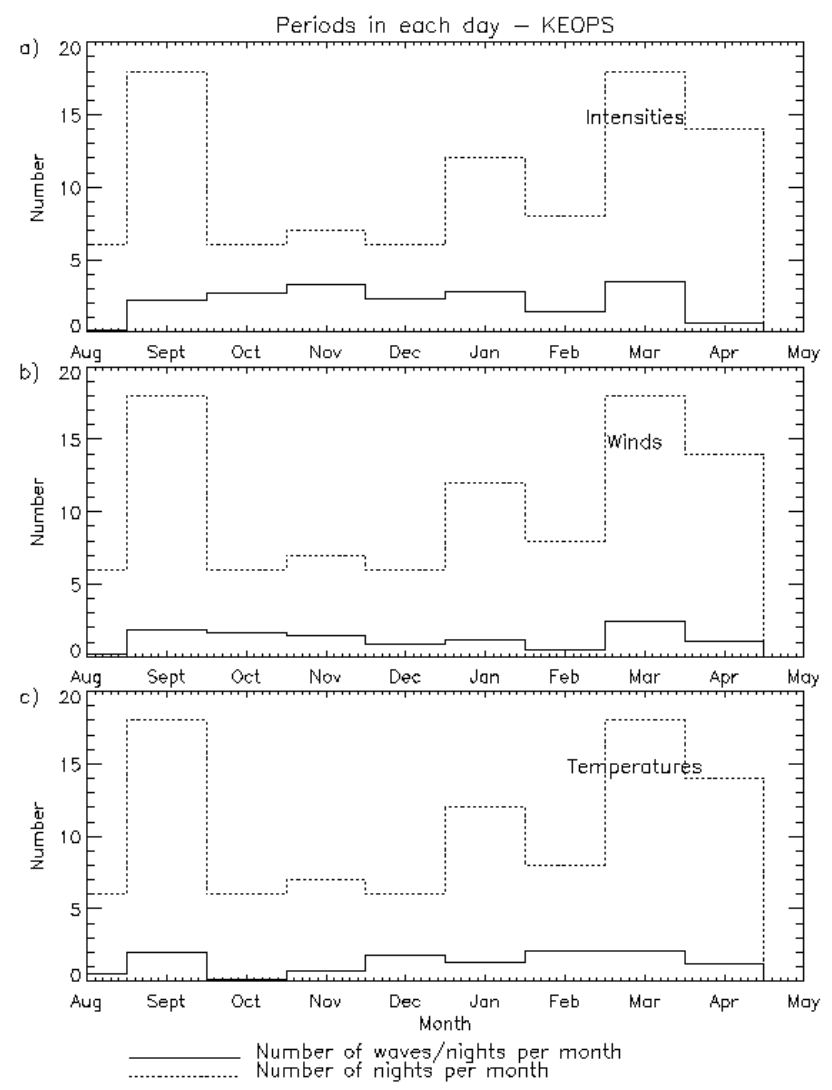

Fig. 15. Monthly counts of gravity waves per number of clear nights, from KEOPS during 2000-2001: (a) intensities, (b) winds, and (c) temperatures.

shows that there were an increased number of clear nights in September, March, and April compared with the other months. The solid line shows the number of gravity waves detected in each month, over $4 \mathrm{~h}$, and above the $70 \%$ confidence level, divided by the number of nights used in that month. An increased number of waves are seen in March where the equinox occurs, in the intensities and winds. The vernal equinox in September sees an increase in the number of waves in the winds and temperatures, but this is shifted to November in the intensities. Nights in August and much of April are too short to detect many waves. The numbers of waves seen within these limits are too low to draw any 

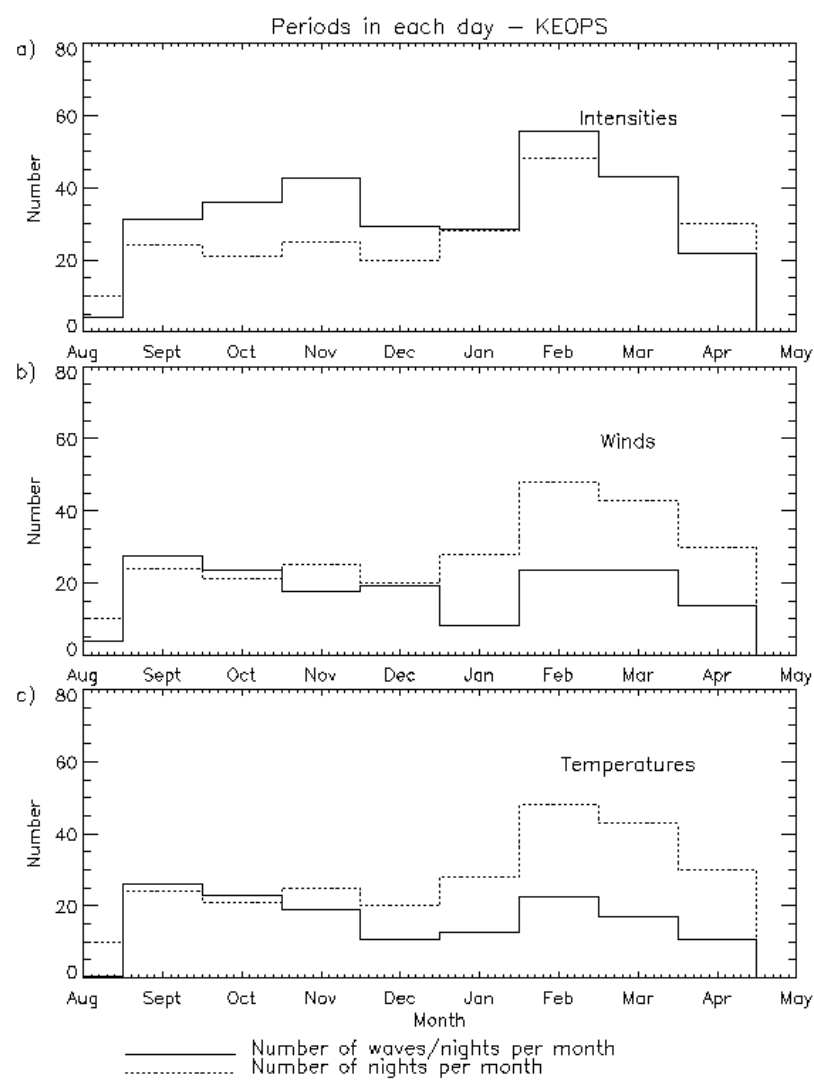

Fig. 16. Number of gravity waves with periods $0-4 \mathrm{~h}$ detected in each month as a proportion of the number nights of data, for all KEOPS data (2000-2006): (a) intensities, (b) winds, and (c) temperatures.

statistical conclusions; however, an increase in the numbers of waves around the equinoxes is possible. This can be seen using all the years of data, from Fig. 8.

Figure 16 shows the KEOPS data for 2000-2006 using periods less than $4 \mathrm{~h}$ in order to remove the bias introduced by the seasonal variation of the length of night, in the same format as Fig. 15. Waves are counted for each look direction individually (resulting in greater than 31 counts per month). The number of clear nights (dotted line) shows that spring weather conditions are the best. The KEOPS intensities in Fig. 16a show statistically significant peaks in the number of waves detected in November and February, and for the winds and temperatures this is nearer September and February. There is a minimum around December and January, at the winter solstice. The standard error of the mean is 3.9, 3.0 and 2.8 for the intensities, winds and temperatures, respectively. There is a statistically significant (i.e. greater than the standard error on the mean) increase in the number of gravity waves at the equinoxes. A Students t-test for this data gives a $\mathrm{P}$ value of 0.0535 for the intensities, showing that this is nearly at the $95 \%$ confidence (5\% significance level). For the intensities, the peaks in Fig. 16 are more towards mid-
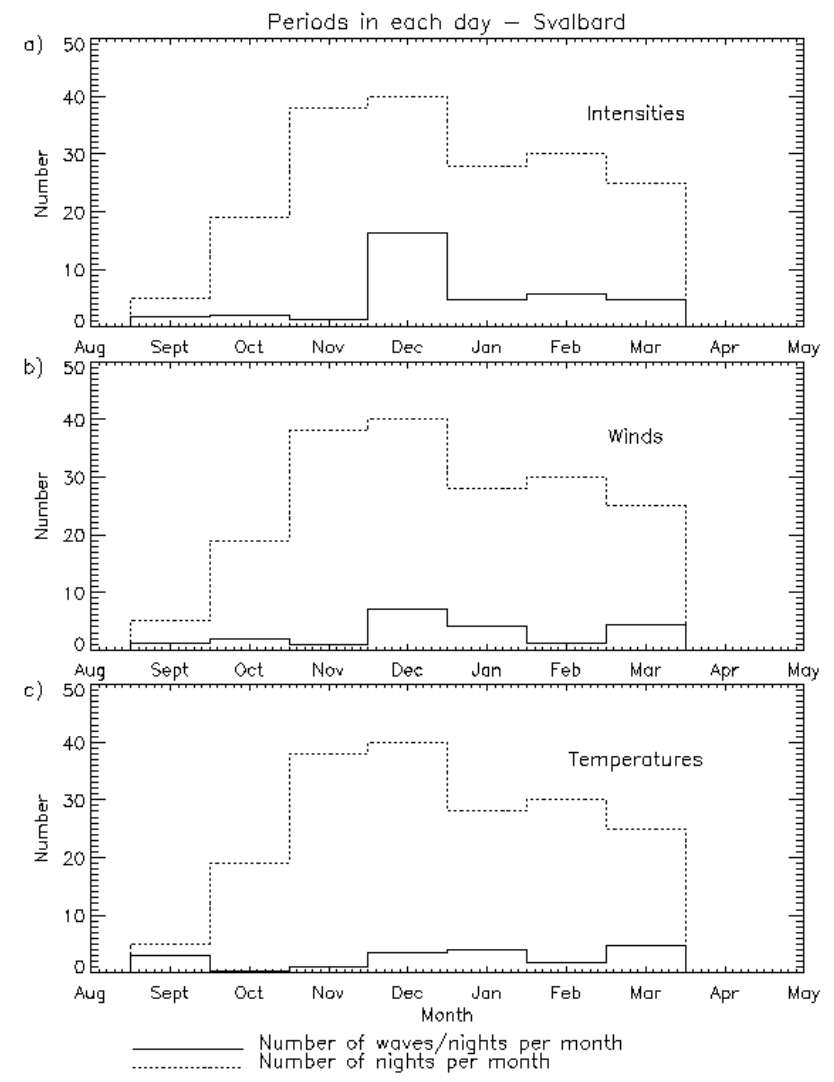

Fig. 17. Number of gravity waves with periods $0-4 \mathrm{~h}$ detected in each month as a proportion of the number nights of data, for all Svalbard data (2000-2006): (a) intensities, (b) winds, and (c) temperatures.

winter than the other parameters, so November data are included as equinox for the intensities, but not the winds and temperatures, as for the 2000-2001 season data in Fig. 15. The $\mathrm{P}$ values from the $\mathrm{t}$-test for these are 0.0092 and 0.0174 , respectively. These are over the $95 \%$ confidence level and therefore are significant results.

Geomagnetic activity peaks around the equinoxes (e.g. McIntosh, 1959), due to the orientation of the Earth's magnetic field with respect to the Interplanetary Magnetic Field, so this could explain the increased number of waves here. However, compositional changes may also affect the number of gravity waves formed. For example, the winter anomaly produces a peak in electron density in mid-winter. This may explain why the peaks in Fig. 16 are not at the equinoxes, as would be expected if it was just a geomagnetic activity dependence, but that the peaks are nearer the winter solstice.

Figure 17 shows the Svalbard data in the same format as Fig. 16. Here is shown a statistically significant peak in the number of waves per night in the middle of winter in the intensities and winds. (The standard errors of the means are $3.5,2.5$ and 2.1 for the intensities, winds and temperatures, respectively). This may indicate a dependence on 
composition, i.e. the winter anomaly which causes a larger electron density in winter, which thereby will increase ionneutral coupling. The consequence of this difference between KEOPS and Svalbard is that the propagation of the waves polewards may be more efficient at mid-winter.

\subsection{Solar cycle variations}

Comparisons between the data in Fig. 4 from 2005-2006 and Fig. 14 from 2000-2001 should show any solar cycle dependences. In these latter years, the solar cycle has been approaching its minimum, and this can be seen from the increased proportion of geomagnetically quiet nights compared with 2000-2001. This effect can also be seen in the Sodankylä and Svalbard data that are shown later. However, despite there being more nights with moderate and high geomagnetic activity levels in the 2000-2001 data, there are no more gravity waves at any period bin in Fig. 14 than in Fig. 4. There is still generally only one wave in active nights in any one period bin, and at most two. Although the numbers of active nights are low so the results are inconclusive, it appears that changes in solar output has little effect on the gravity waves produced. This would be in agreement with, for example, Ogawa et al. (1987), who proposed that gravity waves are relatively easily formed in the thermosphere and large amounts of precipitation or energy input are not required to produce the gravity waves. This was, however, from ionospheric data from a range of lower thermospheric altitudes, so may not be applicable to the FPI data.

A significant difference between the 2000-2001 data (Fig. 14) and the 2005-2006 data (Fig. 4) is that the latter period shows a large number of short period $(<2 \mathrm{~h})$ gravity waves. This is not likely to be a solar cycle effect, but the result of different time resolutions due to different detectors. The 2000-2001 had a cycle period of $15 \mathrm{~min}$ compared with $3.5 \mathrm{~min}$ in 2005-2006. This was discussed above with respect to Fig. 5. This lack of solar cycle variation means that the difference in data collection period between KEOPS (2000-2006) and Sodankylä (2002-2006) will not affect the comparisons made when looking at the different years together, for example in Sect. 3.7. The main advantage to KEOPS having data from earlier years is to increase the statistical significance of the results.

\section{Conclusions}

Data from the FPIs at KEOPS, Sodankylä, and Svalbard on all the clear nights from the years 2000 to 2006 have been analysed for gravity wave activity. A total of 249 nights were available from KEOPS, where data were collected through the night and the skies were clear. The Sodankylä and Svalbard FPIs provided 133 and 185 nights of data, respectively. 567 nights of data have therefore been used in this study. A Lomb-Scargle analysis was performed on each of these nights, as for the case studies described in Ford et al. (2006, 2007), to identify the periods of any wave activity during the night. Comparisons between many nights of data allow the general characteristics of the waves that are present in the high latitude upper thermosphere to be determined.

No particular individual frequencies are preferred in the thermospheric gravity waves detected by the FPIs; though a wave with a period of $6 \mathrm{~h}$ was seen is some datasets which was either an atmospheric tide or due to the two cell convection pattern in the polar cap. The distribution of the number of waves across the period range (approximately $20 \mathrm{~min}$ to $8 \mathrm{~h}$ ), however, was not uniform. The time resolution of the data was the most important factor in the number of short period waves detected, and slow cycle times determine both the shortest period that can be detected and reduce the spectral power of the lowest period waves. The numbers of long period waves that are detected are limited by the length of the night. However, in the 2004-2005 KEOPS dataset, where all the nights were longer than $16 \mathrm{~h}$, there were few waves with periods greater than approximately 3 to $4 \mathrm{~h}$, implying gravity waves have preferred periods in the intensities shorter than $4 \mathrm{~h}$.

For the wind and temperature data there is a more even distribution with respect to period, of the numbers of waves than for the intensities. Also longer periods, up to around $5 \mathrm{~h}$, were more common. The differences are thought to be due to the response times of the different parameters to particle precipitation. The intensities seem to react very quickly to the energy input from bursts of precipitation, but there is an inertia within the winds and temperatures due to the bulk of the thermosphere, which acts as a low pass filter, decreasing the number of short period waves formed.

Comparing data from different sites from the same year, and so at the same point in the solar cycle, but with different time resolutions, allowed comparisons of how the resolution affects the periods of waves found. The distribution of periods from low-resolution intensities had a closer correlation to that of the wind and temperature data than that of the high-resolution intensity data from later years. This implies that the intensities give a closer indication of the distribution of periods than the winds and temperatures, as these do not show the short period waves.

Comparisons of datasets from KEOPS at solar maximum and solar minimum did not show any change in the number of waves produced. This implies solar activity has little effect on the production of gravity waves, possibly because gravity waves are relatively easily formed in the thermosphere and do not require high geomagnetic activity. However, the number of nights available for this solar cycle study was not large, and so this result is inconclusive.

Differences in the gravity wave properties due to geomagnetic activity levels cannot be easily determined from one season of data, due to the low numbers of active nights. Therefore, several years of data were used to determine this dependence. Significant statistical samples were nonetheless 
only created with the KEOPS data. The total nights were separated into geomagnetically quiet $\left(K_{p} \leq 3\right.$ all through the night), moderate activity levels ( $3 \leq K_{p} \leq 6$ all night), and active nights $\left(K_{p}>7\right.$ at some time in the night). This, however, did not allow any statistically significant difference to be shown between the different activity levels in the distribution of the gravity waves over the period range for any of the sites for the intensities, winds, or the temperatures.

Seasonal variations were studied by counting the number of waves detected in each day of the year, for a year when there was a reasonably even distribution of clear nights of data across the year (at KEOPS in 2000-2001). The change in distribution of waves over the period range over the year is mainly determined by the varying length of the night, so that more long period waves are detected in the mid-winter months when longer data sets are available. Within the uncertainties in timings, the wave periods have a reasonably even distribution. However, binning the days of observations into months for the total of the 5 winters indicates a small geomagnetic activity dependence. A slightly larger number of waves are seen around the equinoxes for KEOPS, due to a peak in geomagnetic activity around the equinoxes. At Svalbard a peak is seen near mid-winter, likely due to compositional changes affecting electron density. This could mean that the propagation of the waves polewards may be more efficient at mid-winter.

KEOPS data from the 2004-2005 season was used to compare for each period bin, waves found in the four cardinal look directions separately with the proportion of waves found in any one of the look directions,. This was used to find that, on average, a wave is seen, at a confidence level above $70 \%$, in $60 \%$ of the look directions. This shows that in over half the cases, the wave is close to the site of KEOPS, so auroral sources are likely, as the nominal auroral oval is just to the north of KEOPS. When waves are not seen in all of the look directions, information on the propagation of the gravity waves may be deduced, and can be due to several reasons. The wave could dissipate part way through the field of view of the FPI. This implies either a source location further away, which will only be the case on very geomagnetically quiet nights, or a weak wave. A wave may have a source within the FPI field of view and so propagates in only one direction. Due to the small distances between the look directions in relation to the typical wavelengths of the gravity waves, it is more likely that gravity waves propagate away from the source in a non-uniform direction. This is likely to be in an equatorward or poleward direction, perpendicular to the auroral oval.

Comparisons between the number of gravity waves detected at KEOPS and Sodankylä over all the seasons showed a similar proportion of waves to the number of nights used for both sites, at periods outside the influence of the time resolution of the data and the length of the nights. This was as expected as the two sites are at similar latitudes and therefore locations with respect to the auroral oval, confirming this as a likely source region. Comparisons between the Svalbard data with those from KEOPS and Sodankylä again showed the effects of the time resolution of the datasets to the distribution of periods of waves detected. Svalbard, at a higher latitude, has more nights which have long hours of darkness, hence more long period gravity waves are detected. Svalbard and Sodankylä over the total range of seasons have similar average time resolutions, and both sites have a reduced number of short period waves in relation to KEOPS, which has a much shorter average time resolution.

However, comparisons between data sets with the same time resolution showed differences between the sites, and Svalbard showed fewer waves with short periods (less than an hour) than the KEOPS data for the same season (20052006). Energy is dissipated through Joule heating in each cycle of a wave, therefore, over a given distance, short period waves lose more energy and dissipate. This gives a clear indication of the direction of flow of the gravity waves, and corroborates that the source is the auroral oval and waves propagate polewards to the polar cap.

Acknowledgements. The FPIs are funded and maintained through PPARC grant PPA/G/O/2001/00484. The ESRANGE KEOPS facility, the Sodankylä Geophysical Observatory and LAPBIAT grant, and UNIS in Svalbard have provided generous help in logistics.

Topical Editor U.-P. Hoppe thanks one anonymous referee for her/his help in evaluating this paper.

\section{References}

Aruliah, A. L. and Griffin, E. M.: Evidence of meso-scale structure in the high latitude thermosphere, Ann. Geophys., 19, 37-46, 2001, http://www.ann-geophys.net/19/37/2001/.

Aruliah, A. L., Griffin, E. M., McWhirter, I., Aylward, A. D., Ford, E. A. K., Charalambous, A., Kosch, M. J., Davis, C. J., and Howells, V. S. C.: First tristatic studies of meso-scale ion-neutral dynamics and energetics in the high-latitude upper atmosphere using collocated FPIs and EISCAT radar, Geophys. Res. Lett., 31, L03802, doi:10.1029/2003GL018469, 2004.

Aruliah, A. L., Griffin, E. M., Aylward, A. D., Ford, E. A. K., Kosch, M. J., Davis, C. J., Howells, V. S. C., Pryce, E., Middleton, H., and Jussila, J.: First direct evidence of meso-scale variability on ion-neutral dynamics co-located tristatic FPIs and EISCAT radar in Northern Scandinavia, Ann. Geophys., 23, 147162,2005 , http://www.ann-geophys.net/23/147/2005/.

Born, M. and Wolf, E.: Principles of Optics, 6th edition, Oxford, Pergamon Press, 1987.

de Deuge, M. A., Greet, P. A., and Jacka, F.: Optical observations of gravity waves in the auroral zone, J. Atmos. Terr. Phys., 56, 617-629, 1994.

Ford, E. A. K., Aruliah, A. L., Griffin, E. M., and McWhirter, I.: Thermospheric gravity waves in Fabry-Perot Interferometer measurements of the $630.0 \mathrm{~nm}$ OI line, Ann. Geophys., 24, 555566, 2006, http://www.ann-geophys.net/24/555/2006/. 
Ford, E. A. K., Aruliah, A. L., Griffin, E. M., and McWhirter, I.: High time resolution measurements of the thermosphere from Fabry-Perot Interferometer measurements of atomic oxygen, Ann. Geophys., 25, 1267-1278, 2007, http://www.ann-geophys.net/25/1267/2007/.

Fritts, D. C. and Alexander, M. J.: Gravity Wave dynamics and effects in the middle atmosphere, Rev. Geophys., 41, 1003, doi:10.1029/2001RG000106, 2003.

Fritts, D. C. and Hoppe, U. P.: High-Resolution Measurements of Vertical Velocity with the European Incoherent-Scatter VHF Radar 2: Spectral Observations and Model Comparisons, J. Geophys. Res., 100, 16827-16838, 1995.

Hargreaves, J. K.: The Upper Atmosphere and Solar - Terrestrial Relations, Berkshire, Van Nostrand Reinhold Co. Ltd., 1979.

Hecht, E.: Optics, 4th edition, Addison Wesley, 2001.

Hernandez, G.: Fabry-Perot Interferometers, Cambridge, Cambridge University Press, 1986.

Hocke, K. and Schlegel, K.: A review of atmospheric gravity waves and travelling ionospheric disturbances: 1982-1995, Ann. Geophys., 14, 917-940, 1996,

http://www.ann-geophys.net/14/917/1996/.

Hocke, K.: Phase estimation with the Lomb-Scargle periodogram method, Ann. Geophys., 16, 356-358, 1998, http://www.ann-geophys.net/16/356/1998/.

Hunsucker, R. D.: Atmospheric gravity waves generated in the high-latitude ionosphere: a review, Rev. Geophys. Space Phys., 20, 293-315, 1982.

Innis, J. L. and Conde, M.: High-latitude thermospheric vertical wind activity from Dynamics Explorer 2 Wind and Temperature Spectrometer observations: Indications of a source region for polar cap gravity waves, J. Geophys. Res., 107, 1172, doi:10.1029/2001JA009130, 2002.
Innis, J. L. and Conde, M.: Thermospheric vertical wind activity maps derived from Dynamics Explorer-2 WATS observations, Geophys. Res. Lett., 28, 3847-3850, 2001.

Innis, J. L., Greet, P. A., and Dyson, P. L.: Thermospheric gravity waves in the southern polar cap from 5 years of photometric observations at Davis, Antarctica, J. Geophys. Res., 106, 15489 $15500,2001$.

Kosch, M. J., Hagfors, T., and Rees, D.: A new Fabry-Perot interferometer for atmospheric studies with the EISCAT incoherent radar, Adv. Space Res., 20, 1133-1136, 1997.

Lomb, N. R.: Least squares frequency analysis of unequally spaced data, Astrophys. Space Sci., 39, 447-462, 1976.

McIntosh, D. H.: On the Annual Variation of Magnetic Disturbance, Phil. Trans. Royal Soc., A251, 525-552, 1959.

Ogawa, T., Igarashi, K., Aikya, K., and Maeno, H.: NNSS satellite observations of medium scale travelling ionospheric disturbances at southern high latitudes, J. Geomagnetism and Geoelectricity, 39, 709-721, 1987.

Press, W. H. and Rybicki, G. B.: Fast algorithm for spectral analysis of unevenly sampled data, Astrophys. J., 338, 277-280, 1989.

Scargle, J. D.: Studies in Astronomical time series analysis: II Statistical aspects of spectral analysis of unevenly spaced data, Astrophys. J., 263, 835-853, 1982.

Solomon, S. C., Hays, P. B., and Abreu, V. J.: The auroral 6300A emission: observations and modeling, J. Geophys. Res., 93, 9867-9882, 1988.

Williams, P. J. S., Virdi, T. S., Lewis, R. V., Lester, M., Rodger, A. S., McCrea, I. W., and Freeman, K. S. C.: Worldwide atmospheric gravity-wave study in the European sector 1985-1990, J. Atmos. Terr. Phys., 55, 683-696, 1993. 